\title{
THE PROGENITOR DEPENDENCE OF THE PRE-EXPLOSION NEUTRINO EMISSION IN CORE-COLLAPSE SUPERNOVAE
}

\author{
Evan O'CONnOR ${ }^{1}$ and Christian D. OtT ${ }^{1,2,3}$ \\ ${ }^{1}$ TAPIR, California Institute of Technology, Mailcode 350-17, Pasadena, CA 91125, USA; evanoc@ tapir.caltech.edu, cott@ tapir.caltech.edu \\ 2 Kavli IPMU, University of Tokyo, Kashiwa, Japan \\ Received 2012 July 4; accepted 2012 November 16; published 2012 December 21
}

\begin{abstract}
We perform spherically symmetric general-relativistic simulations of core collapse and the postbounce pre-explosion phase in 32 presupernova stellar models of solar metallicity with zero-age main-sequence masses of $12-120 M_{\odot}$. Using energy-dependent three-species neutrino transport in the two-moment approximation with an analytic closure, we show that the emitted neutrino luminosities and spectra follow very systematic trends that are correlated with the compactness $(\sim M / R)$ of the progenitor star's inner regions via the accretion rate in the pre-explosion phase. We find that these qualitative trends depend only weakly on the nuclear equation of state (EOS), but quantitative observational statements will require independent constraints on the EOS and the rotation rate of the core as well as a more complete understanding of neutrino oscillations. We investigate the simulated response of water Cherenkov detectors to the electron antineutrino fluxes from our models and find that the large statistics of a galactic core collapse event may allow robust conclusions on the inner structure of the progenitor star.
\end{abstract}

Key words: equation of state - hydrodynamics - neutrinos - stars: evolution - stars: neutron - supernovae: general

Online-only material: color figures

\section{INTRODUCTION}

The radial instability of the electron-degenerate, Chandrasekhar-mass core marks the beginning of the final episode in the life of a massive star with zero-age main-sequence (ZAMS) mass in the range $\sim 8-130 M_{\odot}$. Collapse ensues and, once fully dynamical, separates the core into a subsonically homologously contracting inner core and a supersonically collapsing outer core. At nuclear density, the repulsive component of the nuclear force leads to a stiffening of the equation of state (EOS). This stabilizes the inner core, which overshoots its new equilibrium, then rebounds into the outer core, launching a strong hydrodynamic shock wave from its edge. This instant in time is referred to as core bounce. The inner core has a mass of $\sim 0.5 M_{\odot}$ at bounce and this material becomes the unshocked core of the protoneutron star. The shock formed at core bounce propagates into the outer core, but the dissociation of nuclei in the accreted material into neutrons and protons and the electron capture on free protons in the region behind the shock (the postshock region) soon sap its might, driving it into submission to the ram pressure of accretion. The shock stalls and turns into a standing accretion shock that must be revived to unbind the stellar envelope and drive a core-collapse supernova explosion.

Neutrinos play a pivotal and dominant role in stellar collapse and core-collapse supernovae. Neutrinos and antineutrinos of all flavors carry away the $\sim 300 \mathrm{~B}\left(=3 \times 10^{53} \mathrm{erg}\right)$ of gravitational binding energy of the remnant neutron star over tens of seconds after core bounce. Aided by multi-dimensional fluid instabilities, they probably deposit, within a few hundred milliseconds after bounce, sufficient energy in the region behind the stalled shock to revive the shock and cause a typical $\sim 1 \mathrm{~B}$ core-collapse supernova explosion (Janka et al. 2007; Müller et al. 2012b, and references therein). Only hyper-energetic (i.e., $\mathcal{O}(10) \mathrm{B}$ ) explosions may require a different mechanism (Ugliano et al. 2012), e.g., rapid rotation combined with strong magnetic fields, which

\footnotetext{
3 Alfred P. Sloan Research Fellow.
}

may lead to energetic jet-driven explosions (e.g., Burrows et al. 2007).

For a galactic or near-extragalactic core-collapse supernova, neutrinos offer the unique possibility of directly observing the dynamics and thermodynamic conditions prevalent in the supernova core. Together with gravitational waves (see, e.g., Ott 2009; Kotake 2011) they will herald the next nearby supernova possibly hours before any telescope sensitive to electromagnetic waves will notice the event. In the probable case that the next galactic supernova occurs in a dust-enshrouded region and/or close or behind the galactic center, the supernova may be impossible to observe in broadbands of the electromagnetic spectrum, making neutrino and gravitational-wave observations even more important.

The observation of neutrinos from SN 1987A in the Large Magellanic Cloud (Hirata et al. 1987; Bionta et al. 1987; Alekseev et al. 1987) confirmed the basic picture of core collapse and early protoneutron star evolution (e.g., Sato \& Suzuki 1987; Bruenn 1987; Burrows \& Lattimer 1986; Burrows 1987, 1988; Arnett et al. 1989; Jegerlehner et al. 1996; Loredo \& Lamb 2002; Yüksel \& Beacom 2007; Pagliaroli et al. 2009b, and references therein), but the small number and poor timing of the observed interactions did not allow far-reaching and robust conclusions on core-collapse supernova dynamics and the involved neutrino physics, nuclear physics, and astrophysics.

The situation will be completely different when the neutrino burst from a galactic core-collapse supernova reaches current and near-future neutrino detectors on Earth. SuperKamiokande (Fukuda et al. 2003; Ikeda et al. (SuperKamiokande Collaboration) 2007), IceCube (Abbasi et al. (IceCube Collaboration) 2011), LVD (Aglietta et al. 1992; Vigorito (LVD Collaboration) 2011), Borexino (Alimonti et al. (BOREXINO Collaboration) 2009; Cadonati et al. 2002), KamLAND (Piepke 2001), SNO+ (Kraus \& Peeters 2010), Nova (Davies (for the NOvA Collaboration) 2011), and others will together see many thousands of neutrinos from a core collapse event at $10 \mathrm{kpc}$ (Scholberg 2012). Distance estimates 
based on the observed neutrino flux (Kachelrieß et al. 2005), sky localization (Beacom \& Vogel 1999; Tomas et al. 2003), and triggering of gravitational-wave searches by the reconstruction of the time of core bounce (Pagliaroli et al. 2009a; Halzen \& Raffelt 2009) will likely all be possible.

Well-timed high-statistics coincident neutrino observations will allow us to probe in detail a broad range of supernova astrophysics, nuclear physics, and neutrino physics (see Burrows et al. 1992; Wurm et al. 2012; Raffelt 2010 for overviews). Fast characteristic temporal variations in the pre-explosion neutrino fluxes would be tell-tale signs of multi-dimensional fluid instabilities in the postshock region (Ott et al. 2008; Marek et al. 2009; Lund et al. 2010; Brandt et al. 2011) and/or early postbounce ring-down oscillations of a rapidly spinning protoneutron star (Ott et al. 2012). While artificially driven, spherically symmetric core collapse simulations suggest that a sudden deep drop of the accretion-driven component of the neutrino luminosity (primarily in $v_{e}$ and $\bar{v}_{e}$ ) within a few hundred milliseconds would indicate the onset of explosion (e.g., Burrows et al. 1992; Fischer et al. 2010, 2012), the situation is likely different in nature as multidimensional simulations show a much shallower drop due to simultaneous accretion and explosion of material. This leads to a persistent accretion luminosity even after the explosion has begun (Müller et al. 2012a, 2012b). The spectral characteristics and long-term spectral evolution of the neutrino flux could provide important constraints on the nuclear EOS (Roberts et al. 2012; Marek et al. 2009) and/or the spin of the progenitor core (Ott et al. 2008; Marek \& Janka 2009). The ability of some detectors to distinguish interactions of different neutrino flavors would lead to constraints on the neutronto-proton ratio in the neutrino-driven wind phase, allowing an observational test of core-collapse supernovae as potential sites for $r$-process nucleosynthesis (Hüdepohl et al. 2010; Fischer et al. 2010; Wurm et al. 2012).

The neutrino signature of core collapse, of the subsequent core-collapse supernova evolution, and of the protoneutron star cooling phase, is invariably intertwined with neutrino oscillation physics. The robustness of all of the above-mentioned observational conclusions will depend on our understanding of the impact of neutrino flavor oscillations. Neutrinos propagating from their emission site to detectors on Earth may experience (1) so-called vacuum oscillations driven by neutrino mass differences (Pontecorvo 1968), (2) oscillations mediated by a resonance in $v-e^{-}$scattering (the Mikheyev-Smirnov-Wolfenstein (MSW) effect; Mikheev \& Smirnov 1986; Wolfenstein 1978), and (3) oscillations due to $v-v$ scattering (Pantaleone 1992; see Duan et al. 2010 for a review). Vacuum and MSW oscillations are well understood and their outcomes depend essentially only on neutrino mixing parameters, in particular the neutrino mass hierarchy and the mixing angles. The $v-v$-scattering-driven oscillations, on the other hand, have a nonlinear Hamiltonian that may lead to so-called collective oscillations with very complex spatial and temporal outcome that remains to be fully understood (see, e.g., Hannestad et al. 2006; Duan et al. 2007, 2010; Fogli et al. 2009; Dasgupta \& Dighe 2008, and references therein). However, a number of recent studies suggest that collective oscillations may be completely or at least partially suppressed in the pre-explosion accretion phase of ordinary core-collapse supernovae (Chakraborty et al. 2011a, 2011b; Sarikas et al. 2012), but see Cherry et al. (2012) and Dasgupta et al. (2012) for discrepant results.

Provided that collective oscillations can be ignored in the preexplosion phase and that the $\theta_{13}$ mixing angle indeed has the large value suggested by recent measurements (An et al. 2012), the neutrino mass hierarchy may be inferred from the qualitative shape of the early postbounce neutrino signal (Kachelrieß et al. 2005; Serpico et al. 2012).

A pre-explosion accretion phase with suppressed collective oscillations would also offer the opportunity to probe the structure of the progenitor star on the basis of the observed neutrino signal. The details of the pre-explosion neutrino emission have been discussed carefully, e.g., by Thompson et al. (2003) and Liebendörfer et al. (2004) and we shall not repeat them here. It is, however, necessary to outline its most salient features. For simplicity, we neglect neutrino oscillations in the following.

In core collapse and in the subsequent postbounce evolution, emission of $v_{e}$ and $\bar{v}_{e}$ occurs via charged and neutral currents, while heavy-lepton neutrinos $v_{x}=\left\{v_{\mu}, \bar{v}_{\mu}, v_{\tau}, \bar{v}_{\tau}\right\}$ are created exclusively via thermal neutral-current pair processes. Before core bounce, only $v_{e}$ are emitted from electron capture in the collapsing core. Milliseconds after core bounce, the shock breaks out of the $v_{e}$ neutrinosphere (where the optical depth is $\tau_{v_{e}} \approx 2 / 3$ ) and a strong burst of $v_{e}$ is emitted for $\sim 20 \mathrm{~ms}$ from rapid electron capture on the freshly abundant free protons behind the shock. $v_{x}$ are copiously created in the hot interior of the protoneutron star after bounce and begin to diffuse out, leading to a steep rise, quick leveling and subsequent slow decay of the $v_{x}$ luminosity $\left(L_{v_{x}}\right) . \bar{v}_{e}$ production via charged-current positron capture is initially suppressed due to the high degeneracy of the electrons. The latter is partially lifted after bounce at the moderate-density, hot edge of the protoneutron star and $L_{\bar{v}_{e}}$ rises, reaching or surpassing the value at which $L_{v_{e}}$ levels off after the neutronization burst decays. The subsequent pre-explosion luminosity can roughly be split into a diffusive component from the core and accretion luminosity ( $\propto G M\left[R_{v}\right] \dot{M} / R_{v}$, where $R_{v}$ is an approximate neutrinosphere radius) from or from above the neutrinosphere (Burrows 1988). $L_{v_{x}}$ is primarily diffusive, while $L_{v_{e}}$ and $L_{\bar{v}_{e}}$ are dominated by accretion. In general, $L_{v_{e}} \approx L_{\bar{v}_{e}}>L_{v_{x}}$, but $4 L_{v_{x}}=L_{v_{\mu}}+L_{\bar{v}_{\mu}}+L_{v_{\tau}}+L_{\bar{v}_{\tau}}>L_{v_{e}}+L_{\bar{v}_{e}} \cdot v_{x}$ have the lowest opacity, since they interact only via neutral currents. They decouple from matter at the smallest radii and highest temperatures and thus have the highest average energies $\left\langle\epsilon_{v}\right\rangle$. $\bar{v}_{e}$ have a slightly lower opacity than $v_{e}$, leading to the wellestablished neutrino energy hierarchy in the pre-explosion phase $\left\langle\epsilon_{v_{x}}\right\rangle>\left\langle\epsilon_{\bar{v}_{e}}\right\rangle>\left\langle\epsilon_{v_{e}}\right\rangle$. The mean energy of all species grows with increasing postbounce time, reflecting the recession of the neutrinospheres due to the contraction of the protoneutron star.

Considering that the accretion luminosity will scale with the postbounce accretion rate $\dot{M}$, one would naturally expect an increase of the detected neutrino interactions with increasing mass of the stellar core. Since higher accretion rates correspond to more material compressing and settling more rapidly on the protoneutron star, the latter's outer regions will be hotter. Thus, the thermal neutral-current emission will be enhanced, leading to higher luminosities and higher mean neutrino energies.

The variation of the pre-explosion neutrino signal with progenitor star ZAMS mass was first discussed by Woosley et al. (1986) based on the pioneering simulations of Wilson (1985) and Wilson et al. (1986). These authors provided total emission characteristics and spectra that show a systematic increase of total energy emitted in neutrinos and mean $\bar{v}_{e}$ energy with ZAMS mass in the range from 10 to $25 M_{\odot}$. Mayle et al. (1987), before SN 1987A, carried out simulations of a range of progenitor stars with ZAMS mass in the range $12-100 M_{\odot}$. They found that the $v_{e}$ neutronization burst shows 
little dependence on the progenitor, due to the rather universal homologous collapse and bounce dynamics. Furthermore, they mentioned, though did not discuss in detail, that the luminosities and mean neutrino energies increase as a function of iron core mass (and not ZAMS mass). A more detailed and clear physical discussion was provided by Bruenn (1987), who contrasted the predicted neutrino signal from the early postbounce phase in two different progenitor core models with neutrino observations of SN 1987A. He noted that there are significant uncertainties in connecting a given ZAMS mass to precollapse structure. Instead of a progenitor with an associated ZAMS mass, he considered a massive (and high-entropy) $2.05 M_{\odot}$ iron core model and a lower-mass (and lower-entropy) $1.35 M_{\odot}$ iron core model in his spherically symmetric (1D) neutrino radiation-hydrodynamic simulations. He showed that the more massive core leads to a consistently higher $\bar{v}_{e}$ luminosity in both the accretion and diffusion sectors. The water Cherenkov detectors that observed neutrinos from SN 1987A are most sensitive to the inverse beta decay (IBD) reaction $\bar{v}_{e}+p \rightarrow n+e^{+}$. Bruenn (1987) predicted a factor-of-two difference in the integrated number of early IBD interactions between the massive and the lowmass core in these detectors. He concluded that the neutrino signal observed by these detectors from SN 1987A was most consistent with the low-mass core. Burrows (1988), who carried out a parameter study of quasi-hydrostatic protoneutron star cooling, considering various initial masses, ad hoc accretion rates, and different nuclear EOS, found a similar trend. He showed that more massive cores, higher accretion rates, and softer EOS lead to stronger, higher-energy neutrino emission. Some of his strongest emitters were cases in which eventually a black hole was formed.

Liebendörfer and collaborators carried out a sequence of studies of the progenitor dependence of the neutrino signal using modern general relativistic $1 \mathrm{D}$ radiation-hydrodynamics simulations (Liebendörfer et al. 2001, 2002, 2003, 2004). They showed that the $v_{e}$ neutronization burst is indeed almost independent of progenitor structure (as first suggested by Mayle et al. 1987). They also qualitatively and quantitatively connected the evolution of the postbounce pre-explosion luminosity to the postbounce accretion rate, but did not discuss observational implications. Their results were corroborated by similarly sophisticated subsequent studies of Kachelrieß et al. (2005), Buras et al. (2006), Fischer et al. (2009, 2010, 2012), Sumiyoshi et al. (2008), and Serpico et al. (2012). Of these, Buras et al. (2006) presented the most comprehensive analysis and also compared between $1 \mathrm{D}$ and axisymmetric (2D) results. They found that in $2 \mathrm{D}$, convection in the protoneutron star alters the structure of the latter, affecting the neutrino emission starting $\sim 100 \mathrm{~ms}$ after bounce, but preserving the overall systematics with accretion rate. Buras et al. (2006) also were the only authors to suggest that the accretion-rate dependence of luminosity and total emitted energy in the pre-explosion phase could be used to infer the structure of the progenitor. The other studies, being focused on aspects such as neutrino oscillations, black hole formation, or the late-time post-explosion evolution, did not consider observational consequences.

Thompson et al. (2003), using a limited set of three progenitor models $\left(\{11,15,20\} M_{\odot}\right.$ at ZAMS $)$, found similar systematics as the aforementioned studies, but also carried out an analysis of the expected signal in various neutrino detectors in the first $250 \mathrm{~ms}$ after bounce. They computed IBD interaction rates for their $20 M_{\odot}$ and $11 M_{\odot}$ and found a factor of two more IBD interactions for the former, which would allow a high-confidence distinction between these progenitors for a galactic core collapse event. However, their $15 M_{\odot}$ model yielded a postbounce neutrino signal very similar to that of their $11 M_{\odot}$ model and would be indistinguishable by neutrino observations alone. This suggests that ZAMS mass is not a good parameter to describe presupernova stellar structure (cf. Bruenn 1987).

In this paper, we present a fresh look at the progenitor dependence of the neutrino signature in the pre-explosion accretion phase of core-collapse supernovae. We perform 1D general relativistic radiation-hydrodynamics core collapse simulations of 32 progenitor models from the single-star solar-metallicity presupernova model suite of Woosley \& Heger (2007) and follow the postbounce pre-explosion evolution for $450 \mathrm{~ms}$. In ZAMS mass, these models range from $12 M_{\odot}$ to $120 M_{\odot}$, but guided by the previous results discussed in the above, we choose not to parameterize our simulations by ZAMS mass. Instead we employ the compactness parameter $\xi_{M} \sim M / R(M)$ (for a relevant mass scale $M$, measured at the time of bounce). As shown in O'Connor \& Ott (2011), and further explored in Ugliano et al. (2012), $\xi_{M}$ is a quantitative stellar structure parameter that describes the postbounce accretion evolution to a remnant mass scale $M$. We demonstrate that the pre-explosion neutrino emission is very well parameterized by the compactness. The pre-explosion luminosities and mean energies of all neutrino species increase essentially monotonically with increasing $\xi_{M}$. We compute predicted integrated IBD interactions for a galactic core-collapse supernova in the Super-Kamiokande detector and show that the clear systematics governed by $\xi_{M}$ carries over to observation, even when standard MSW neutrino oscillations are taken into account. Our results thus indicate that - in the absence of complicated collective neutrino oscillations-a highstatistics detection of neutrinos from the pre-explosion phase will allow, in principle, a tight constraint of the compactness of the progenitor star's core. This, however, will require knowledge of the nuclear EOS and of the rotation rate of the collapsed core, since, as we show, both can dilute the otherwise clear compactness-dependent neutrino emission systematics.

This paper is structured as follows. In Section 2, we discuss our general relativistic hydrodynamics code GR1D and introduce its extension to neutrino radiation hydrodynamics in the twomoment approximation, nuGR1D. The initial models and the employed EOS are discussed in Section 3. In Section 4, we present results from a benchmark collapse and postbounce simulation that allows us to compare with the previously published code comparison of Liebendörfer et al. (2005) to assess nuGR1D's ability to reproduce results of full Boltzmann neutrino transport. We present the results of our simulations in Section 5, analyze the dependence of the neutrino signal on progenitor compactness, discuss predicted IBD signals from a galactic core collapse event in the Super-Kamiokande detector, and explore potential degeneracies introduced by EOS and rotation. Finally, in Section 6, we critically summarize our work and conclude by contrasting our results with the early neutrino signal observed from SN 1987A.

\section{METHODS}

We make use of the open-source 1D general relativistic hydrodynamics code GR1D (O'Connor \& Ott 2010; available at http://www.stellarcollapse.org) outfitted with an energydependent multi-species M1 neutrino transport scheme in which the zeroth and first moments of the neutrino distribution function are evolved. We refer the reader to O'Connor \& Ott (2010) 
for details on GR1D and describe in the following our current implementation of the transport scheme. For this first application, we neglect the computationally expensive energy-coupling neutrino interactions and transport terms-these terms are undoubtedly important for making highly accurate predictions of the neutrino signature (see, e.g., Lentz et al. 2012a, 2012b), but are unlikely to affect the general trends we observe. We will address them in future work, but provide a discussion on the consequences of neglecting these terms via a comparison to full Boltzmann neutrino transport simulations in Section 4.

Our M1 scheme closely follows Shibata et al. (2011), who formulate the M1 evolution equations in a closed covariant form. The scheme is simplified greatly by neglecting the energy-coupling terms. This further requires that the velocitydependent terms are also ignored. In this limit, and using the Schwarzschild-like metric and radial-polar slicing of GR1D and setting $G=c=M_{\odot}=1$, the coordinate frame evolution equations for the neutrino energy density, $E_{(v)}$, and the neutrino flux vector, $F_{r,(v)}$, simplify from Equations (3.37) and (3.38) of Shibata et al. (2011) to

$$
\partial_{t} E_{(v)}+\frac{1}{r^{2}} \partial_{r}\left(\frac{\alpha r^{2}}{X^{2}} F_{r,(v)}\right)=\alpha^{2} \mathcal{S}_{(v)}^{t}
$$

and

$$
\partial_{t} F_{r,(v)}+\frac{1}{r^{2}} \partial_{r}\left(\frac{\alpha r^{2}}{X^{2}} P_{r r,(v)}\right)=\alpha X^{2} \mathcal{S}_{(v)}^{r}+\alpha \frac{E_{(v)}\left(1-p_{(v)}\right)}{r},
$$

where $\mathcal{S}^{\alpha}$ is the neutrino interaction source term (see below), $\alpha$ is the lapse function and $X=(1-2 M(r) / r)^{-1 / 2} . P_{r r,(v)}$ is the neutrino pressure tensor and is taken to be an interpolation between the two limiting cases of free streaming and diffusion. We follow Shibata et al. (2011), who express $P_{i i,(v)}$ as

$$
P_{i i,(v)}=\frac{3 p_{(v)}-1}{2} P_{i i,(v), \text { thin }}+\frac{3\left(1-p_{(v)}\right)}{2} P_{i i,(v), \text { thick }},
$$

where $p_{(v)}$ is the Eddington factor, taken here to be the maximum entropy closure in a closed, analytic form (Minerbo 1978; Cernohorsky \& Bludman 1994),

$$
p_{(v)}=\frac{1}{3}+\frac{f_{(v)}^{2}}{15}\left(6-2 f_{(v)}+6 f_{(v)}^{2}\right) .
$$

In the no-velocity limit for GR1D, $f_{(v)}=\left|F_{r,(v)} /\left(E_{(v)} X\right)\right|$. The free streaming and diffusion limits of the neutrino pressure tensor are $P_{r r,(v), \text { thin }}=E_{(v)} X^{2}$ and $P_{i i,(v), \text { thick }}=g_{i i} E_{(v)} / 3$, respectively.

We set out to solve the system of equations via standard hyperbolic methods borrowed from conservative hydrodynamic schemes (Pons et al. 2000). Transport variables live at cell centers and we employ piecewise linear reconstruction to cell interfaces with van Leer's limiter (van Leer 1977) and the HLLE approximate Riemann solver (Einfeldt 1988) for calculating the intercell fluxes. A complication arises when solving the neutrino moment equations in the high-opacity limit. In this case, the standard fluxes returned from the Riemann solver are dominated by a numerical diffusion term. We follow Audit et al. (2002) and modify the fluxes to correct for this. Essentially, the modified fluxes return the diffusion-limit flux in the high opacity limit. The modified form of the neutrino energy density flux through the $i+1 / 2$ interface is given by

$$
F_{r,(v)}^{i+1 / 2}=\frac{\tilde{a}^{+} F_{r,(v)}^{i, R}-\tilde{a}^{-} F_{r,(v)}^{i+1, L}+\epsilon_{(v)} \tilde{a}^{+} \tilde{a}^{-}\left(E_{(v)}^{i+1, L}-E_{(v)}^{i, R}\right)}{\tilde{a}^{+}-\tilde{a}^{-}} .
$$

The corresponding modified interface flux for the neutrino flux evolution equation is

$$
P_{r r,(v)}^{i+1 / 2}=\epsilon_{(v)} \tilde{P}_{r r,(v)}^{i+1 / 2}+\left(1-\epsilon_{(v)}^{2}\right)\left(P_{(v)}^{i+1, L}+P_{(\nu)}^{i, R}\right) / 2,
$$

with

$$
\tilde{P}_{r r,(v)}^{i+1 / 2}=\frac{\epsilon_{(v)}\left(\tilde{a}^{+} P_{r r,(v)}^{i, R}-\tilde{a}^{-} P_{r r,(v)}^{i+1, L}\right)+\tilde{a}^{+} \tilde{a}^{-}\left(F_{(\nu)}^{i+1, L}-F_{(v)}^{i, R}\right)}{\tilde{a}^{+}-\tilde{a}^{-}} .
$$

In these equations, $\epsilon_{(v)}$ controls the modification to the fluxes to account for the high opacity. Following Audit et al. (2002), we take

$$
\epsilon_{(v)}=\min \left(1, \frac{1}{\kappa_{(v)} \Delta r}\right),
$$

where $\kappa_{(v)}$ is the sum of the scattering and absorptive opacities. These opacities are strong functions of energy and are also species dependent. We note that when $\epsilon_{(v)}$ is 1 , the intercell fluxes reduce to the standard HLLE approximation. The characteristic speeds needed for the HLLE scheme are calculated in the same spirit as the neutrino pressure tensor (Shibata et al. 2011; Kuroda et al. 2012),

$$
\lambda_{(v)}=\frac{3 p_{(v)}-1}{2} \lambda_{(v), \text { thin }}+\frac{3\left(1-p_{(v)}\right)}{2} \lambda_{(v), \text { thick},}
$$

where in the zero velocity limit, $\lambda_{(v) \text {,thin }}= \pm \alpha / X$ and $\lambda_{(v) \text {,thick }}=$ $\pm \alpha /(\sqrt{3} X) . \tilde{a}^{+}$and $\tilde{a}^{-}$are the maximum and minimum values, respectively, of these characteristic speeds evaluated from both the right and left reconstructed variables.

Finally, the source terms in Equations (1) and (2) are taken from Shibata et al. (2011). In the zero velocity limit,

$$
\begin{gathered}
\mathcal{S}^{t}=\left(\eta_{(v)}-\kappa_{a,(v)} E_{(v)}\right) / \alpha, \\
\mathcal{S}^{r}=-\left(\kappa_{a,(v)}+\kappa_{s,(v)}\right) F_{r,(v)} / X^{2},
\end{gathered}
$$

where $\eta_{(v)}, \kappa_{a,(v)}$, and $\kappa_{s,(v)}$ are the neutrino emissivity, neutrino absorption opacity, and the neutrino scattering opacity, respectively. We precompute the neutrino interaction terms for each neutrinos species (we treat $v_{e}, \bar{v}_{e}$ and $v_{x}=\left\{v_{\mu}, \bar{v}_{\mu}, v_{\tau}, \bar{v}_{\tau}\right\}$ ) and neutrino energy group in dense tabular form as a function of density $\rho$, temperature $T$, and electron fraction $Y_{e}$. We then use linear interpolation for efficient on-the-fly interpolation. We include all standard iso-energetic scattering processes, chargedcurrent absorption and emission, and thermal pair-production processes (Burrows et al. 2006; Bruenn 1985) in the calculation of the neutrino interaction terms. Since the neutrino-matter interactions for heavy-lepton neutrinos and antineutrinos are slightly different, NuLib averages the two values of the emissivities and opacities. Our library of neutrino interaction routines, which we call NuLib, is open source and available as a GitHub repository at http://www.nulib.org. NuLib requires an EOS for the evaluation of the emissivities and opacities. Our treatment of thermal pair processes in GR1D warrants some comments. 
Since we do not currently consider energy (or species) coupling for thermal emission processes such as electron-positron annihilation to a neutrino-antineutrino pair, we compute an emissivity based on the thermal content of the matter ignoring any final state neutrino blocking. To limit the neutrino energy density to the equilibrium value (where neutrino-antineutrino annihilation rates are in equilibrium with the thermal pair production rates), we use Kirchhoff's law to derive an effective absorption opacity for neutrino-antineutrino annihilation from the thermal emissivity,

$$
\kappa_{a,(v)}^{\text {eff, thermal }}=\eta_{(\nu)}^{\text {thermal }} / B_{(v)},
$$

where $B_{(v)}=c E_{(v)}^{3} /(2 \pi \hbar c)^{3} f_{(v)}^{\mathrm{eq}}$ is the thermal energy density of neutrinos with energy $E_{(v)}$ and $f_{(v)}^{\mathrm{eq}}=1 /\left(\exp \left[\left(E_{(v)}-\mu\right) / T\right]+1\right)$ is the equilibrium neutrino distribution function with chemical potential $\mu$. As we shall see, this method performs well at predicting the thermal neutrino flux of the heavy-lepton neutrinos during the pre-explosion phase.

In nuGR1D, we first update the hydrodynamic variables to the $n+1$ th timestep. We then compute the neutrino opacities and emissivities associated with the updated hydrodynamic variables. We update the radiation field operator split. The flux term is solved explicitly, using the radiation moments of the $n$th timestep. We calculate the neutrino-matter interaction terms using the $n+1$ radiation moments via a local implicit update. With the $n+1$ radiation energy density source term, we then update the energy density and electron fraction of the matter. We use 24 energy groups, with lowest-energy group centers at $0.5 \mathrm{MeV}$ and $1.5 \mathrm{MeV}$, and then spaced logarithmically up to $200 \mathrm{MeV}$ for $v_{e}, \bar{v}_{e}$, and $v_{x}$. We note that for the highest energy bins it occasionally occurs that the evolved neutrino flux vector exceeds the evolved neutrino energy density. This tends to occur in the most dynamic phases of our simulations and where the opacities vary significantly from one zone to the next. When this is the case we limit the neutrino flux to the neutrino energy density. We extract the radiation quantities in the coordinate frame at a radius of $500 \mathrm{~km}$.

\section{INITIAL MODELS AND EQUATIONS OF STATE}

We employ the most recent non-rotating solar-metallicity single-star model set from the stellar evolution code KEPLER (Woosley \& Heger 2007). This model set contains the presupernova configuration of 32 stars ranging in ZAMS mass from $12 M_{\odot}$ to $120 M_{\odot}$. We denote individual models by $s X X \mathrm{WH} 07$, where $X X$ corresponds to the integer ZAMS mass of the model, e.g., $s 12 \mathrm{WH} 07$ is the $12 M_{\odot}$ model of this model set. In O'Connor \& Ott (2011), we investigated this and other model sets in the context of black hole formation. Under the assumption of a failed core-collapse supernova, we found a strong empirical relation between the properties of the presupernova structure and the evolution of the failing supernova, e.g., the time to black hole formation. This led to a clear prediction: if we observe black hole formation in a failed core-collapse supernova via neutrinos, the lifetime of the protoneutron star (and thus of the neutrino signal) relays direct information about the presupernova structure. However, such a prediction (1) requires a failed supernova, which may not be the norm, and (2) has a strong dependence on the nuclear EOS. The empirical parameter introduced in O'Connor \& Ott (2011) is the compactness of the progenitor, measured at the time of core bounce. It is an inverse measure of the radial extent of a given mass coordinate

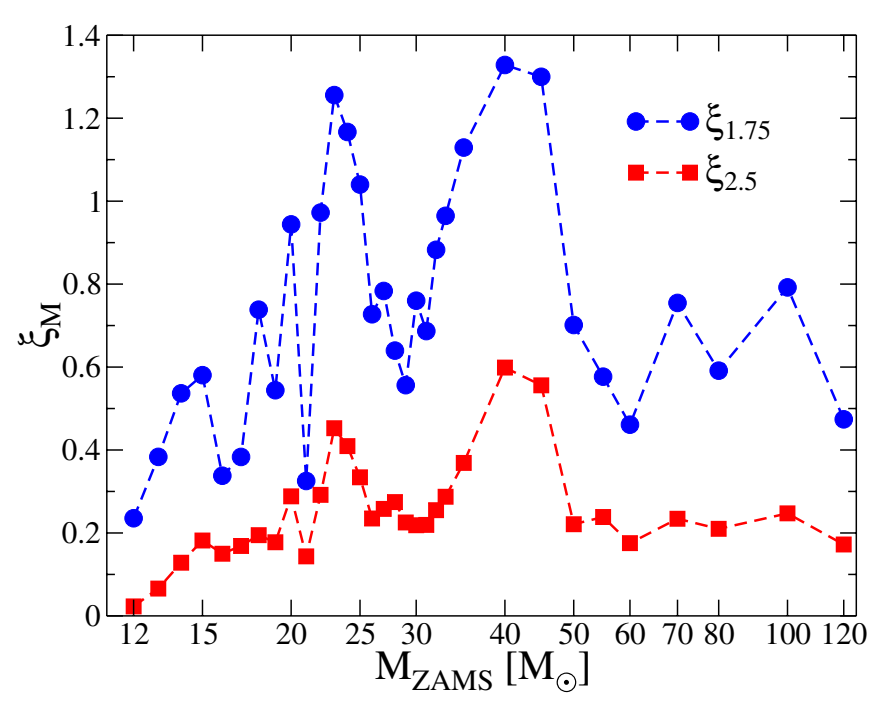

Figure 1. Compactness parameters for the 32 considered presupernova models of Woosley \& Heger (2007) vs. ZAMS mass as evaluated from collapse simulations with the LS220 EOS. We show both $\xi_{1.75}$ and $\xi_{2.5}$. The mapping between ZAMS mass and precollapse structure is highly non-monotonic, making the former an ill-suited parameter for describing progenitor structure in core collapse simulations.

(A color version of this figure is available in the online journal.)

at the time of bounce,

$$
\xi_{M}=\left.\frac{M / M_{\odot}}{R\left(M_{\text {bary }}=M\right) / 1000 \mathrm{~km}}\right|_{t=t_{\text {bounce }}},
$$

where $R\left(M_{\text {bary }}=M\right)$ is the radial coordinate that encloses a baryonic mass of $M$ at the time of core bounce. In O'Connor \& Ott (2011), we chose $M=2.5 M_{\odot}$, since this is the relevant mass scale for black hole formation, i.e., a typical maximum baryonic mass at which a range of EOS can no longer support a neutron star against gravity. In this study, we primarily use $\xi_{1.75}$. The motivation for this is that during the postbounce preexplosion phase, the relevant mass scale, especially for models with relatively small compactness, is much less than $2.5 M_{\odot}$. In this study, we choose $1.75 M_{\odot}$ because this is close to the average baryonic mass inside the shock at 200-300 ms after bounce for all models: in the two extreme models that span the space in compactness parameter (model s12WH07, [ $\xi_{1.75}=0.24$ and $\left.\xi_{2.5}=0.022\right]$, on the lower end; model s40WH07 $\left[\xi_{1.75}=1.33\right.$ and $\left.\xi_{2.5}=0.59\right]$ on the upper end), the baryonic mass accreted through the shock at $250 \mathrm{~ms}$ after bounce is $1.45 \mathrm{M}$ and $2.05 \mathrm{M}$, respectively. We further justify our motivation of using $\xi_{1.75}$ over $\xi_{2.5}$ in Section 5.1. In Figure 1, we plot both $\xi_{1.75}$ and $\xi_{2.5}$ versus ZAMS mass for all 32 considered models. $\xi_{1.75}$ is provided in Table 1 for all models.

For Figure 1, one notes that while $\xi_{1.75}$ and $\xi_{2.5}$ differ quantitatively, there is no significant qualitative difference between them. The overall trends transcending individual models remain, including the two regions of high compactness near 22-25 $M_{\odot}$ and 35-45 $M_{\odot} . \xi_{1.75}$ simply provides a more fine-grained parameterization at the lower mass scale relevant in the first few hundred milliseconds after bounce. Note, however, that there are a few models that have similar $\xi_{2.5}$, but rather different density structure at small enclosed masses and radii and, hence, a different $\xi_{1.75}$. Models s14WH07 and s16WH07 are examples.

In this study, we perform core collapse simulations with each progenitor and two EOS. We use the EOS of Lattimer 
Table 1

Key Neutrino Quantities

\begin{tabular}{|c|c|c|c|c|}
\hline Model & $\xi_{1.75}$ & $\begin{array}{c}E_{400 \mathrm{~ms}}^{v_{e}} / E_{400 \mathrm{~ms}}^{\bar{v}_{e}} / E_{400 \mathrm{~ms}}^{v_{x}} \\
\text { (B) }\end{array}$ & $\begin{array}{c}N_{200 \mathrm{~ms}}^{\mathrm{ibd}} \\
\text { LS220/HShen } \\
\left(10^{3}\right)\end{array}$ & $\begin{array}{c}N_{400 \mathrm{~ms}}^{\mathrm{ibd}} \\
\text { LS220/HShen } \\
\left(10^{3}\right)\end{array}$ \\
\hline$s 12$ & 0.235 & $19.24 / 14.19 / 7.73$ & $1.02 / 0.92$ & $2.13 / 1.78$ \\
\hline$s 13$ & 0.383 & $22.18 / 16.58 / 8.76$ & $1.25 / 1.09$ & $2.53 / 2.07$ \\
\hline$s 14$ & 0.537 & 25.19 / 19.35 / 9.24 & $1.36 / 1.20$ & $3.06 / 2.49$ \\
\hline$s 15$ & 0.580 & $25.51 / 19.59 / 9.17$ & $1.30 / 1.16$ & $3.13 / 2.59$ \\
\hline$s 16$ & 0.338 & $18.91 / 13.72 / 8.20$ & $1.11 / 0.95$ & $2.00 / 1.68$ \\
\hline$s 17$ & 0.383 & 19.93 / 14.54 / 8.57 & $1.20 / 1.02$ & $2.13 / 1.78$ \\
\hline$s 18$ & 0.738 & $28.66 / 22.26 / 10.30$ & $1.55 / 1.36$ & $3.62 / 2.92$ \\
\hline$s 19$ & 0.544 & $23.36 / 17.49 / 9.37$ & $1.43 / 1.23$ & $2.67 / 2.18$ \\
\hline$s 20$ & 0.944 & $29.39 / 22.70 / 11.08$ & $1.79 / 1.55$ & 3.64 / 2.91 \\
\hline$s 21$ & 0.325 & $18.48 / 13.41 / 8.09$ & 1.04 / 0.91 & $1.95 / 1.65$ \\
\hline$s 22$ & 0.972 & $30.06 / 23.29 / 11.26$ & $1.82 / 1.58$ & $3.76 / 3.00$ \\
\hline$s 23$ & 1.256 & $42.00 / 33.88 / 14.68$ & $2.29 / 2.00$ & $5.99 / 4.67$ \\
\hline$s 24$ & 1.167 & 39.14 / 31.35 / 13.66 & $2.16 / 1.89$ & $5.44 / 4.24$ \\
\hline$s 25$ & 1.040 & $32.30 / 25.28 / 11.87$ & $1.93 / 1.68$ & $4.15 / 3.28$ \\
\hline$s 26$ & 0.727 & $24.97 / 18.84$ / 10.10 & $1.59 / 1.38$ & $2.88 / 2.33$ \\
\hline$s 27$ & 0.783 & $25.67 / 19.45$ / 10.21 & $1.61 / 1.40$ & $3.01 / 2.42$ \\
\hline$s 28$ & 0.640 & 25.86 / 19.83 / 9.54 & $1.36 / 1.21$ & $3.16 / 2.62$ \\
\hline$s 29$ & 0.556 & 23.24 / 17.38 / 9.43 & $1.45 / 1.22$ & $2.64 / 2.17$ \\
\hline$s 30$ & 0.760 & $26.61 / 20.30 / 10.19$ & $1.60 / 1.40$ & $3.20 / 2.58$ \\
\hline$s 31$ & 0.687 & 25.23 / 19.09 / 9.95 & $1.56 / 1.35$ & $2.96 / 2.40$ \\
\hline$s 32$ & 0.883 & $28.10 / 21.58 / 10.70$ & $1.71 / 1.49$ & $3.43 / 2.75$ \\
\hline$s 33$ & 0.965 & 30.36 / $23.57 / 11.28$ & $1.82 / 1.58$ & $3.82 / 3.04$ \\
\hline$s 35$ & 1.129 & $35.93 / 28.50 / 12.77$ & $2.06 / 1.79$ & 4.83 / 3.79 \\
\hline$s 40$ & 1.328 & 48.32 / 39.45 / 17.51 & $2.49 / 2.21$ & $7.23 / 5.75$ \\
\hline$s 45$ & 1.300 & 46.42 / $37.81 / 16.56$ & $2.42 / 2.14$ & $6.86 / 5.44$ \\
\hline$s 50$ & 0.701 & $25.60 / 19.42 / 10.00$ & $1.57 / 1.32$ & $3.02 / 2.45$ \\
\hline$s 55$ & 0.577 & 23.56 / 17.65 / 9.52 & $1.48 / 1.25$ & $2.69 / 2.20$ \\
\hline$s 60$ & 0.461 & $21.52 / 15.88 / 9.07$ & $1.33 / 1.12$ & $2.36 / 1.95$ \\
\hline$s 70$ & 0.755 & $25.83 / 19.58 / 10.17$ & $1.60 / 1.39$ & $3.04 / 2.46$ \\
\hline$s 80$ & 0.591 & 23.75 / $17.80 / 9.63$ & $1.48 / 1.26$ & $2.71 / 2.22$ \\
\hline$s 100$ & 0.792 & $29.90 / 23.33$ / 10.59 & $1.61 / 1.42$ & 3.82 / 3.07 \\
\hline$s 120$ & 0.474 & $22.31 / 16.64$ / 9.02 & $1.33 / 1.15$ & $2.52 / 2.07$ \\
\hline
\end{tabular}

Notes. For each model in the Woosley \& Heger (2007) model set we show $\xi_{1.75}$, the cumulative emitted neutrino energy in $v_{e}, \bar{v}_{e}$ and a single $v_{x}$ at $400 \mathrm{~ms}$ after bounce. The numbers correspond to the models run with the LS220 EOS. We also present, for each model, the estimated number of IBD interactions in a Super-Kamiokande-like detector at 200 and $400 \mathrm{~ms}$ after bounce for a supernova at a fiducial galactic distance of $10 \mathrm{kpc}$ for both EOS

\& Swesty (1991) with a nuclear incompressibility of $220 \mathrm{MeV}$. The LS220 EOS is based on a compressible liquid-drop model of the nucleus. Of the publicly available nuclear EOS, the LS220 EOS best matches the constraints from nuclear theory and astrophysical observations (see Figure 1 of Ott et al. 2011 and Demorest et al. 2010; Hebeler et al. 2010; Steiner et al. 2010; Özel et al. 2010). We also employ the relativistic mean field EOS of Shen et al. (2011) that is based on the TM1 parameter set. It is very different from the LS220 EOS. The maximum neutrino-less $\beta$-equilibrium cold neutron star gravitational masses are $2.04 M_{\odot}$ and $2.24 M_{\odot}$ for the LS220 and HShen EOS, respectively. The radius of a neutrino-less $\beta$-equilibrium cold neutron star with a gravitational mass of $1.4 M_{\odot}$ using the LS220 EOS is $12.7 \mathrm{~km}$. For the HShen EOS, the corresponding radius is $14.6 \mathrm{~km}$. For details on our particular implementation and the treatment of the low-density EOS, we refer the reader to O'Connor \& Ott (2010, 2011). The EOS tables, reader, and interpolation routines are available from http://www.stellarcollapse.org.

\section{COMPARISON OF NuLib TO BOLTZMANN TRANSPORT}

Since our implementation of neutrino transport is new and approximate, a comparison with published results of full Boltzmann neutrino transport is warranted. This will allow us to test the ability of our code to reproduce the neutrino luminosities and spectral properties in the pre-explosion phase.

We compare nuGR1D with the results of Liebendörfer et al. (2005), a comparison study between two Boltzmann neutrino transport codes. ${ }^{4}$ The two codes, Agile-BOLTZTRAN (Liebendörfer et al. 2004), and VERTEX (Rampp \& Janka 2002), approach the neutrino transport problem in very different ways. Their results compare very well in the Newtonian limit, but show significant quantitative differences in the general relativistic case. Subsequent modifications to the approximate general relativistic potential used in VERTEX (Marek et al. 2005) have since removed many of the quantitative differences between the codes. The general relativistic test case of Liebendörfer et al. (2005) was the collapse and early postbounce evolution of a $15 M_{\odot}$ (at ZAMS) solar-metallicity progenitor of Woosley \& Weaver (1995), referred to as model $s 15$ WW95 in the following. They employed the LS180 EOS (Lattimer \& Swesty 1991) and a baseline set of neutrino-matter interactions, including coupling of energy groups via inelastic scattering processes (Bruenn 1985; Liebendörfer et al. 2005). We repeat their test here, using the same initial conditions and EOS, with our current approximations and compare the neutrino observables. We emphasize again that the current version of nuGR1D lacks inelastic neutrino-electron scattering and velocity-dependent transport terms. Both are included in the simulations of Liebendörfer et al. (2005). Our transport scheme evolves only the zeroth and first moment of the neutrino distribution function, using an analytic closure to truncate the series of moment equations, whereas Liebendörfer et al. (2005) solve the full Boltzmann equation for neutrino transport.

In Figure 2, we show the luminosities (top panels) and rms energies (bottom panels) of three neutrino species: $v_{e}$ (left panels), and $\bar{v}_{e}$ and $v_{x}$ (right panels). Both the luminosity and rms energies are defined in Liebendörfer et al. (2005), Section 4. The black solid lines are the results obtained with nuGR1D, the red dash-dotted lines are the predictions of Agile-BOLTZTRAN, and the blue dashed lines are the VERTEX results. Overall, the agreement is good; however, there are several systematic differences:

1. The magnitudes of the $v_{e}$ and $\bar{v}_{e}$ luminosities in the early postbounce phase predicted by nuGR1D agree well with the Agile-BOLTZTRAN results but they are systematically lower than the VERTEX results. This discrepancy, which also exists between VERTEX and the Agile-BOLTZTRAN, has been further investigated in Marek et al. (2005) and has since been resolved. The updated VERTEX code employs an improved general relativistic potential and gives comparable amplitudes to Agile-BOLTZTRAN, and hence nuGR1D.

2. The time when the silicon-oxygen interface (located at a baryonic mass coordinate of $1.43 M_{\odot}$ in model $\left.s 15 \mathrm{WW} 95\right)$ accretes through the shock, which is marked by a sharp drop in the $v_{e}$ and $\bar{v}_{e}$ luminosities, is earlier (at $\sim 140 \mathrm{~ms}$ ) in our simulations than in the simulations of Liebendörfer

\footnotetext{
4 The numerical data from this study are available online at http://iopscience.iop.org/0004-637X/620/2/840/fulltext/datafiles.tar.gz.
} 

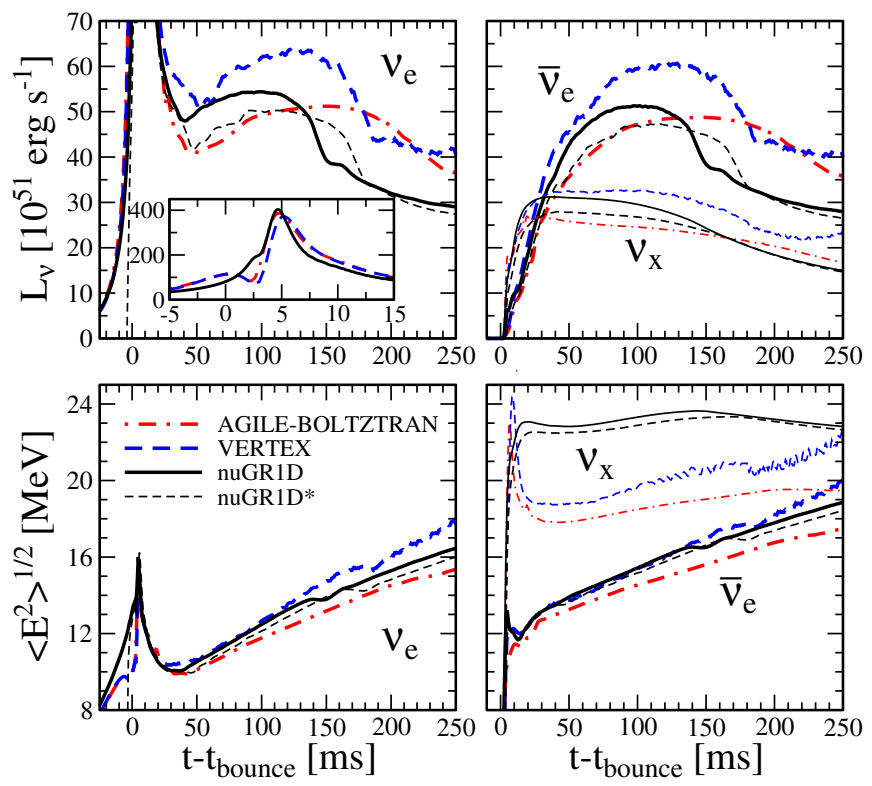

Figure 2. Neutrino luminosities and rms energies plotted as a function of postbounce time for the $s 15 \mathrm{WW} 95$ progenitor. These luminosities (top panels) and energies (bottom panels) correspond to the comparison study of Liebendörfer et al. (2005). The left panels contain results for $v_{e}$, the right panels show $\bar{v}_{e}$ (thick lines) and $v_{x}$ (thin lines) results. The inset plot in the upper left panel shows the $v_{e}$ luminosity around core bounce. Shown in solid black lines are luminosities and rms energies obtained with nuGR1D. The blue dashed lines and red dash-dotted lines are the results from Liebendörfer et al. (2005) using the VERTEX code (Rampp \& Janka 2002) and Agile-BOLTZTRAN code (Liebendörfer et al. 2004), respectively. The thin dashed black lines (labeled as nuGR1D*) are curves from a test simulation using profile data from the VERTEX simulation $5 \mathrm{~ms}$ before bounce as starting data. A detailed discussion of the differences is provided in the text.

(A color version of this figure is available in the online journal.)

et al. (2005) $\left(\sim 180 \mathrm{~ms}\right.$ in the VERTEX simulations $\left.{ }^{5}\right)$. We have carried out a number of tests to try to understand this difference. We have varied the zero point of the internal energy, which affects the relativistic enthalpy entering the momentum and energy equations and alters the collapse time. We have attempted different mappings of the presupernova stellar structure to our Eulerian grid, we have tested variations in precollapse electron capture by parameterizing $Y_{e}$ as a function of density in the collapse phase (Liebendörfer 2005), and we have replaced the low-density compositions with a pure silicon gas (which replaces the NSE equivalent of mainly iron and therefore better represents the low-density matter). None of these tests led to a change of the postbounce time to silicon-oxygen interface accretion by more than $\sim 10 \mathrm{~ms}$.

Without currently having the modeling technology to test it, we suspect that the apparent difference may be due to the only other obvious difference between nuGR1D and VERTEX: our lack of detailed neutrino physics during the collapse phase. Two facts lead us to this conclusion. The collapse time discrepancy between the VERTEX $\left(t_{\text {collapse }} \sim\right.$ $177 \mathrm{~ms}$ ) and Agile-BOLTZTRAN ( $t_{\text {collapse }} \sim 172 \mathrm{~ms}$ ) and nuGR1D $\left(t_{\text {collapse }} \sim 224 \mathrm{~ms}\right)$ can be explained by the lack

\footnotetext{
5 Such a sharp drop is not seen in the Agile-BOLTZTRAN results. Liebendörfer et al. (2005) attribute the lack of a sharp drop in the Agile-BOLTZTRAN results to the use of an adaptive grid, which introduces artificial diffusion and smears out sharp density features. Nevertheless, the slow decline of the $v_{e}$ and $\bar{v}_{e}$ luminosities begins around the same time as in the VERTEX simulations.
}

of inelastic scattering. M. Liebendörfer (2011, private communication) gives a $t_{\text {collapse }} \sim 213 \mathrm{~ms}$ in Agile-BOLTZTRAN when neutrino-electron inelastic scattering is neglected. This collapse time difference, $\sim 40 \mathrm{~ms}$, is the difference in the silicon-oxygen interface accretion times. Also, when we map in the VERTEX profiles from Liebendörfer et al. (2005) at $5 \mathrm{~ms}$ before bounce to nuGR1D and continue the evolution we reproduce the postbounce time that the silicon-oxygen interface reaches the shock. We show the result of this simulation with a thin dashed curve, marked as nuGR1D*, in Figure 2. It is worth noting that there may be additional differences due to the simplistic treatment of the EOS at low densities. Below a density of $6 \times 10^{7} \mathrm{~g} \mathrm{~cm}^{-3}$, VERTEX replaces the nuclear statistical equilibrium (NSE) EOS with an EOS that specifically depends on the composition of the matter. Agile-BOLTZTRAN assumes all matter below this density is silicon. Both have some treatment of nuclear burning. In GR1D, as discussed in O'Connor \& Ott (2010), we assume NSE compositions from the nuclear EOS. At densities below the validity regime of the nuclear EOS, we take the compositions at the lowest density point from the nuclear EOS and use the Timmes EOS (Timmes $\&$ Arnett 1999). For reference, the initial density of the silicon-oxygen interface is $0.4-1.0 \times 10^{7} \mathrm{~g} \mathrm{~cm}^{-3}$, where the range represents the extent in density space.

3. The $v_{e}$ and $\bar{v}_{e}$ rms energies, predicted by nuGR1D agree very well with the Boltzmann transport results during the postbounce phase. The difference seen in the $v_{x}$ rms energy is similar to that observed by Thompson (2002) and Lentz et al. (2012a) when investigating the effects of inelastic neutrino-electron scattering. In the postbounce evolution, this interaction is expected to predominately affect the $v_{x}$ neutrino. We currently ignore this process in nuGR1D and note that the $v_{x}$ luminosity predicted by nuGR1D still agrees well with the full Boltzmann results.

4. Another difference between the evolution in nuGR1D and the full Boltzmann transport results arises in the collapse phase. The lack of velocity terms and inelastic $v_{e}-e^{-}$ scattering significantly effects the composition of the inner core. In simulations with inelastic $v_{e}-e^{-}$scattering, neutrinos from electron capture on free protons down-scatter off of electrons to lower energies. Since the optical depth is lower, these neutrinos can then escape, deleptonizing the core. In our simulations, these high-energy neutrinos cannot down-scatter and therefore cannot escape. Deleptonization is suppressed until later phases, when central density and temperature are higher. The lack of velocitydependent terms delays full trapping by neutrino advection that would normally begin to occur at $\rho \gtrsim 1 \times 10^{12} \mathrm{~g} \mathrm{~cm}^{-3}$ until nuclear densities, allowing for further deleptonization. At bounce the central value of $Y_{e}$ are $\sim 0.22$ compared to $\sim 0.29$ in VERTEX and Agile-BOLTZTRAN. The rms $v_{e}$ energies predicted by nuGR1D are higher during the prebounce phase, because the neutrinos do not experience the down-scattering via inelastic neutrino-electron scattering.

Overall, we find that differences in the inner core structure and composition at bounce do not strongly present themselves in the neutrino signal after the collapse phase. Therefore, for this study, we find the current version of nuGR1D to be acceptable, since our primary focus is the neutrino signal of the pre-explosion accretion phase. We do note, however, that the lack of energy-coupling terms in our 

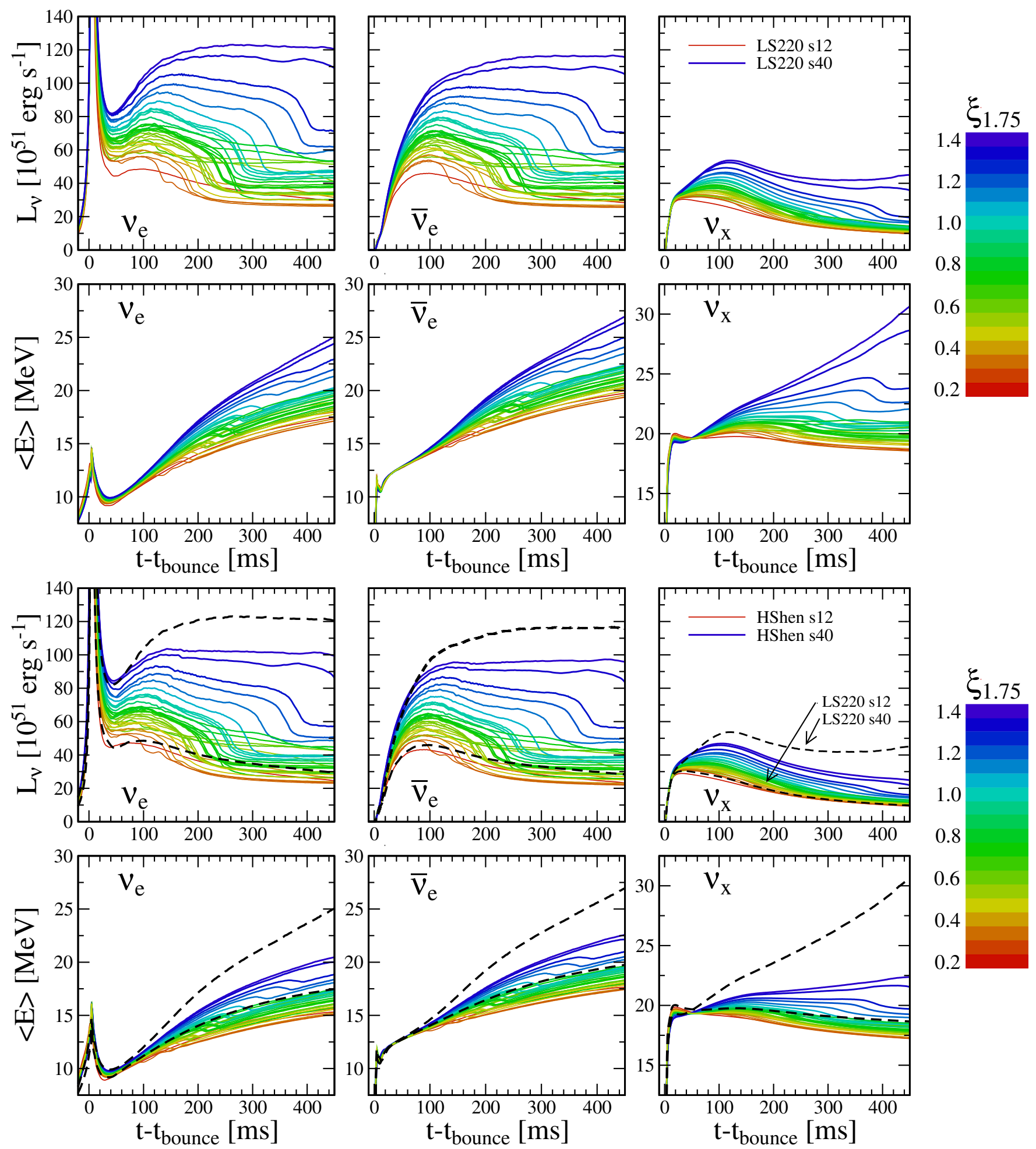

Figure 3. Neutrino luminosities (top panels) and average energies (bottom panels) plotted as a function of postbounce time for all 32 models of Woosley \& Heger (2007). The top set of panels shows results obtained with the LS220 EOS. The bottom panel shows the same for the HShen EOS, but includes, for reference, two LS220 models: $s 12 \mathrm{WH} 07$ and $s 40 \mathrm{WH} 07$. The left, center, and right panels show results for $v_{e}, \bar{v}_{e}$, and $v_{x}$, respectively. The curves are color- and line-weight-coded with increasing compactness $\left(\xi_{1.75}\right)$, the mapping from color to compactness parameter is shown on the right. There is a clear trend in all luminosities and average energies with compactness parameter. The progenitor with the highest compactness, s40WH07, forms a black hole at 503 ms after bounce. None of these models explode, but the onset of an explosion in any of these models may lead to a sudden deep drop (strongest for $\nu_{e}$ and $\bar{v}_{e}$ ) in the luminosities and average energies (Fischer et al. 2010), although this is likely suppressed by multidimensional effects. The smaller drop observed for most models here is due to the sudden decrease of the accretion rate when the silicon-oxygen interface reaches the stalled shock.

(A color version of this figure is available in the online journal.) 
transport can cause qualitative differences near black hole formation. When energy-coupling terms, such as gravitational redshift, are included, then the $v_{e}$ and $\bar{v}_{e}$ luminosities drop off at times very close to black hole formation, as seen in Fischer et al. (2009). This effect can be captured with GR1D's leakage scheme in which it is trivial to include redshift terms (O'Connor \& Ott 2010). Capturing redshift in an energy-dependent transport scheme requires energy-group coupling. We have also compared our M1 scheme to the results of Fischer et al. (2009) for the $40 M_{\odot}$ model from Woosley \& Weaver (1995) using the LS180 EOS. In this model a black hole forms within $500 \mathrm{~ms}$ of bounce. We find differences in the $v_{e}$ and $\bar{v}_{e}$ luminosities of $\sim 10 \%-20 \%$ in the last $\sim 50 \mathrm{~ms}$, but good agreement in the early postbounce phase.

\section{RESULTS}

\subsection{Trends in the Neutrino Observables}

We perform core collapse and early postbounce evolutions of all 32 models introduced in Section 3 using both the LS220 and the HShen EOS. In Figure 3, we present the three neutrino luminosities and average energies for each model and EOS as a function of postbounce time. We do not expect a clear trend in the neutrino observables with ZAMS mass. However, we do expect trends based on the presupernova structure of the star, which is well encapsulated by the compactness parameter introduced in Section 3. In the top set of panels we show simulations run using the LS220 EOS. In the bottom panels, simulations performed with the HShen EOS are shown. To highlight that there is indeed a trend with presupernova structure, we color-code individual models according to their compactness parameter. The mapping between line color and $\xi_{1.75}$ is provided on the right. To more directly highlight the EOS dependence, we include the luminosity and average energies of two models run with the LS220 EOS in the HShen EOS panels with thick dashed lines. These models, $s 12 \mathrm{WH} 07$ and $s 40 \mathrm{WH} 07$, have the lowest and highest compactness parameter in our model set, respectively.

We find that there is little variation in the peak luminosity of the $v_{e}$ neutronization burst signal. For all 32 models simulated using the LS220 (HShen) EOS, the peak amplitude varies by less than $3 \%(5 \%)$ from the average. This reflects the universal nature of the collapse of the inner core (Liebendörfer et al. 2002). After the neutronization burst, the postbounce luminosities of all species increase systematically with increasing compactness parameter. Models with higher $\xi_{1.75}$ have higher temperatures throughout the protoneutron star (O'Connor \& Ott 2011). This increases the diffusive neutrino luminosity and is best seen in the $v_{x}$ luminosities. The postbounce accretion rate also increases with the compactness parameter. Higher accretion rates, and the deeper gravitational potential due to the higher protoneutron star mass, increase the accretion luminosity, which is most directly reflected in the $v_{e}$ and $\bar{v}_{e}$ signals. After roughly $100 \mathrm{~ms}$, the average energy of the emitted neutrinos also shows an increasing trend with the compactness parameter. The matter temperature at the neutrinosphere is higher in models with larger compactness; therefore, a higher average neutrino energy is observed at infinity.

The neutrino luminosities and average energies from simulations using the HShen EOS are systematically lower than the luminosities and average energies from simulations of the same model run with the LS220 EOS. This is clearly seen in the bottom set of panels in Figure 3: models $s 12$ WH07-LS220 and $s$ 40WH07-LS220 have luminosities and average energies that are comparable to or larger than in the corresponding HShen models. For a fixed accretion rate (or fixed progenitor model), the location of the neutrinosphere of each species influences the emitted luminosities and spectra. In models evolved with the stiff HShen EOS, the neutrinospheres are located systematically at larger radii and lower matter temperatures than in models run with the softer LS220 EOS. For example, in the $s 12 \mathrm{WH} 07$ simulations, the Rosseland-mean $v_{e}$ neutrinospheres at $200 \mathrm{~ms}$ after bounce have radii and temperatures of $\sim 35.3 \mathrm{~km}$ and $\sim 4.86 \mathrm{MeV}$; and $\sim 39.5 \mathrm{~km}$ and $\sim 4.46 \mathrm{MeV}$ for the LS220 and the HShen EOS, respectively. The larger neutrinosphere radii are responsible for the lower accretion luminosity since the latter is set essentially by the product of the mass accretion rate and the gravitational potential at the protoneutron star surface (Liebendörfer et al. 2002). The latter is located at larger radii in simulations using the HShen EOS. The differences in the neutrinosphere radii and temperatures between the LS220 and HShen EOS also give an explanation for the systematically lower average neutrino energies seen in the HShen simulations. Matter at larger radii has been compressed less and therefore is cooler. This leads to average neutrino energies that can be up to $5 \mathrm{MeV}$ lower for the HShen EOS than for the LS220 EOS for the same progenitor model (see Figure 3). The difference in the neutrino luminosities and average energies between the two EOS is largest for models with $\xi_{1.75} \gtrsim 1.2$, in which the high accretion rates lead to the accumulation of $\sim 2 M_{\odot}$ of material inside the shock within 200-300 ms of bounce. In the case of the LS220 EOS, this leads to very high temperatures throughout the protoneutron star as it becomes more and more compact and closer to gravitational collapse to a black hole. In our simulations, the most compact model $s 40 \mathrm{WH} 07\left(\xi_{1.75}=1.33, \xi_{2.5}=0.59\right)$ forms a black hole $503 \mathrm{~ms}$ after bounce. The slightly less compact model $s 45 \mathrm{WH} 07\left(\xi_{1.75}=1.30, \xi_{2.5}=0.55\right)$ forms a black hole $563 \mathrm{~ms}$ after bounce. The high temperatures present in the LS220 simulations at these times will not be obtained until postbounce times $\gtrsim 1 \mathrm{~s}$ in models using the HShen EOS. ${ }^{6}$

Most neutrino detectors are most sensitive to the electron antineutrino luminosity through the dominant IBD interaction, $\bar{v}_{e}+p \rightarrow n+e^{+}$. In the left panel of Figure 4, we consider the cumulative emitted $\bar{v}_{e}$ energy for each model using the LS220 EOS. We color code the models based on their compactness parameter and include two reference models that use the HShen EOS, model $s 12 \mathrm{WH} 07$, and model $s 40 \mathrm{WH} 07$. The graphs shown in this panel are the integral of the graphs shown in the top center panel of Figure 3. It is obvious that the cumulative amount of $\bar{v}_{e}$ energy emitted during the pre-explosion phase strongly correlates with the compactness of the progenitor model. For example, the amount of emitted $\bar{v}_{e}$ energy from model $s 40$ WH07 $\left(\xi_{1.75}=1.33\right)$ is always between two and three times of that of model $s 12 \mathrm{WH} 07\left(\xi_{1.75}=0.24\right)$. We make this point more quantitative in the center and right panels of Figure 4. In the center (right) panel we plot the cumulative emitted $\bar{v}_{e}$ energy at 100,200,300, and $400 \mathrm{~ms}$ after bounce for both EOS as a function of $\xi_{1.75}\left(\xi_{2.5}\right)$. For reference, we present a subset of these numbers in Table 1 . We see a very clear correlation that depends only weakly on the chosen EOS. Note, however, that for models with small compactness parameter

\footnotetext{
6 While we do not follow these models to black hole formation in our current study, in O'Connor \& Ott (2011) we found that the black hole formation times of the $s 40 \mathrm{WH} 07$ and $s 45 \mathrm{WH} 07$ models are $\sim 1.3 \mathrm{~s}$ and $\sim 1.4 \mathrm{~s}$, respectively, when using the HShen EOS.
} 

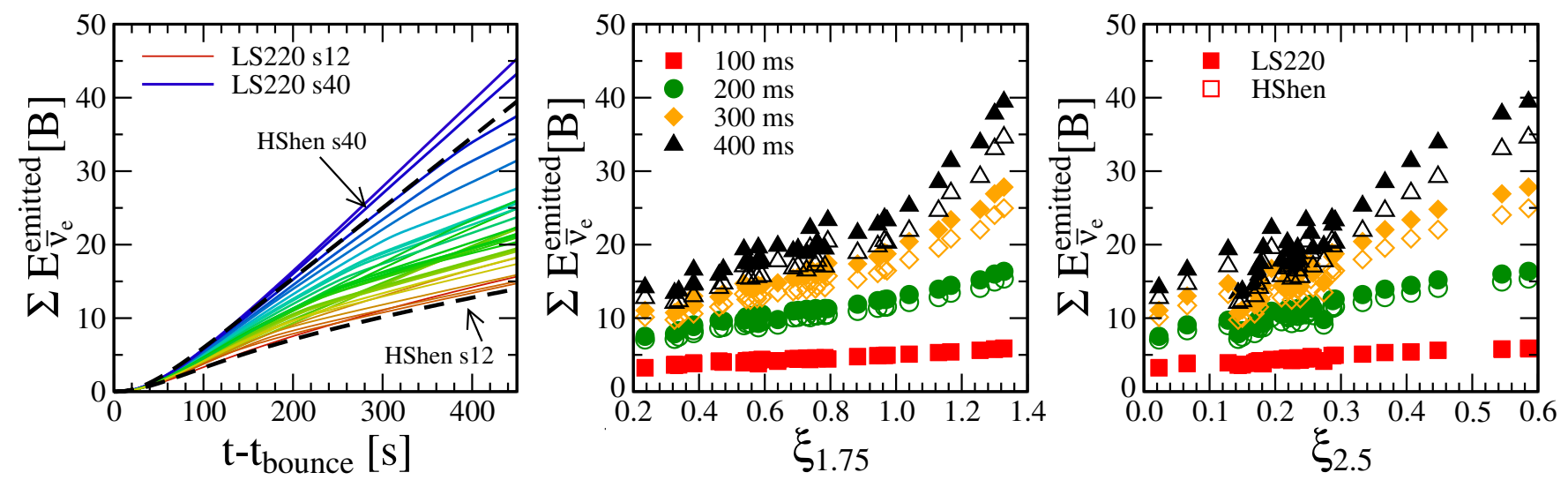

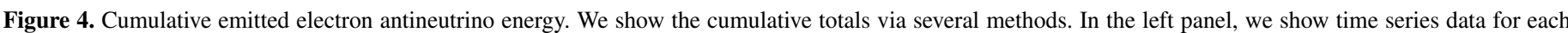

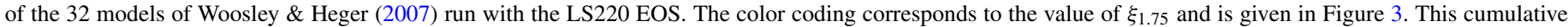

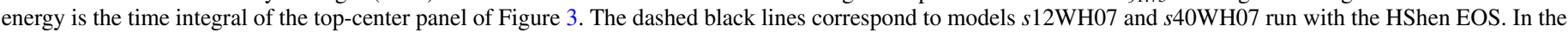

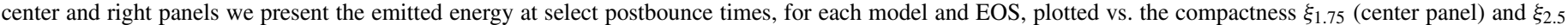
(right panel) of the model.

(A color version of this figure is available in the online journal.)

$\left(\xi_{1.75} \lesssim 0.8\right)$, the correlation between the total emitted $\bar{v}_{e}$ energy and the compactness parameter is not as strong after $400 \mathrm{~ms}$ of postbounce evolution. Comparing the center and right panels justifies our choice of $\xi_{1.75}$ over $\xi_{2.5}$ as explained in Section 3.

The onset of an explosion will break the correlation observed in Figure 4. Once it is launched, the accretion luminosity effectively turns off and only the diffusion luminosity remains. One also expects this diffusion luminosity to show a correlation with the compactness of the progenitor, since the remnant protoneutron star's thermodynamic conditions, such as the central entropy and its mass, are essentially set by the presupernova structure. However, it is currently unclear whether one should obtain a correlation between explosion time and the compactness parameter. Clarification will require a more complete understanding of the core-collapse supernova explosion mechanism and may require extensive parameter studies with fully self-consistent three-dimensional simulations.

\subsection{Detectability}

We use the publicly available software SNOwGLoBES ${ }^{7}$ to predict the neutrino signal observed in Earth-based neutrino detectors. SNOwGLoBES (Beck et al. 2011; Scholberg 2012), which in turn relies on GLoBES (Huber et al. 2005, 2007), is a set of routines that compute the interaction rates of supernova neutrinos in user-specified detector configurations. Variables include detector material (e.g., water, scintillator, argon, and lead), detector volume, detector response functions, and a host of relevant neutrino interactions. For this investigation, we consider only IBD interactions in a water Cherenkov detector. We choose a detector mass of $32 \mathrm{kT}$, the mass of water in Super-Kamiokande that is sensitive to core-collapse supernova neutrinos (Scholberg 2012). For reference, we use the wc100kt30prct smearing rates and efficiencies provided with SNOwGLoBES. We construct SNOwGLoBES initial fluence data from our simulations binned in $5 \mathrm{~ms}$ intervals. We provide these energy-dependent fluences at http://www.stellarcollapse.org/M1prog for all models, neutrino species, and both EOS in $5 \mathrm{~ms}$ intervals up to $450 \mathrm{~ms}$ after bounce. We assume a fiducial galactic supernova distance of $10 \mathrm{kpc}$. In Figure 5, we show the cumulative number of interactions for each model run with the LS220 EOS

\footnotetext{
7 Available at http://www.phy.duke.edu/ schol/snowglobes.
}

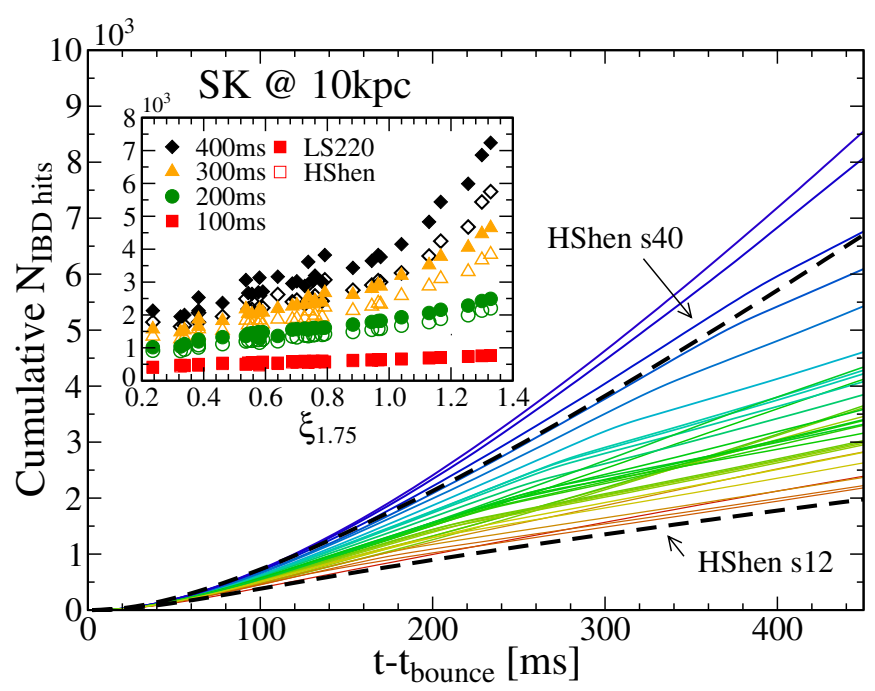

Figure 5. Cumulative IBD interactions in a Super-Kamiokande-like water Cherenkov detector at a fiducial galactic distance of $10 \mathrm{kpc}$ vs. postbounce time. We use the SNOwGLoBES package to determine the integrated IBD interaction rate in a $32 \mathrm{kT}$ water Cherenkov detector at $10 \mathrm{kpc}$. The color coding corresponds to the value of $\xi_{1.75}$ and is provided in Figure 3. The dashed lines are results for models $s 12 \mathrm{WH} 07$ and $s 40 \mathrm{WH} 07$ run with the HShen EOS. In the inset we show the cumulative IBD interactions as a function of $\xi_{1.75}$ for each model and EOS at four postbounce times: 100, 200, 300, and $400 \mathrm{~ms}$.

(A color version of this figure is available in the online journal.)

and, for reference, two models run with the HShen EOS. The lines are color-coded according to compactness parameter. Note that the vertical scale in this figure is in thousands of interactions. We note that our answers agree with the total number of expected detected interactions in Super-Kamiokande from a galactic core-collapse supernova at $10 \mathrm{kpc}$, which is estimated to be $\sim 7000$ (Scholberg 2012). To arrive at this number from our results, consider the lowest ZAMS mass progenitor in our model set, model $s 12 \mathrm{WH} 07$. After $450 \mathrm{~ms}$ of evolution, $15 \mathrm{~B}$ of $\bar{v}_{e}$ energy has been radiated (Figure 4), which corresponds to 2000 IBD detected interactions (Figure 5). For $50 \mathrm{~B}$ of released energy $(\sim 1 / 6$ of $300 \mathrm{~B}$, the fiducial energy released in neutrinos over the entire cooling phase), one would then expect $\sim 7000$ detected interactions. However, as is clear from Figure 5, the number of detected interactions from the next galactic 
supernova may be higher than this fiducial number. More importantly, the rate of interactions in the pre-explosion phase will give us detailed information on the progenitor core structure.

In order to more directly quantify the differences between variations in progenitor compactness and variations in the nuclear EOS, we plot in the inset of Figure 5 the number of expected IBD detected interactions in a Super-Kamiokandelike water Cherenkov detector at various postbounce times versus $\xi_{1.75}$. There is a well-defined trend: the number of IBD interactions detected in the first 100, 200, 300, and $400 \mathrm{~ms}$ increases with the compactness parameter of the models. For reference, we include the expected number of interactions at 200 and $400 \mathrm{~ms}$ for both EOS in Table 1. We find that the EOS dependence of the expected number of interactions is similar to the EOS dependence of the total emitted $\bar{v}_{e}$ energy: the HShen EOS leads to a lower number of interactions (compare the inset of Figure 5 to the center panel of Figure 4). The dependence on EOS is somewhat stronger here, since the lower average $\bar{v}_{e}$ energy predicted from stiffer EOS translates into a reduced cross section in Earth-based detectors. In addition to the total number of interactions, a water Cherenkov detector measures individual energies, and thus, allows for the reconstruction of the cumulative emitted $\bar{v}_{e}$ energy over time. This reconstruction will depend on the detector's response function and efficiency.

An additional independent path to experimentally probing the inner structure of the progenitor is via the total neutrino energy emitted in all species over the first 10 s of seconds after the initial collapse. This method requires a measurement of the total fluence of neutrinos of all species, not just electron antineutrinos. Examples of neutrino interactions capable of relaying such information are the mono-energetic de-excitation of a neutral-current neutrino excitation of ${ }^{12} \mathrm{C}$ (Scholberg 2012) or neutrino-proton elastic scattering interactions (Dasgupta \& Beacom 2011). Such measurements would require good energy resolution, a significant source of carbon and/or a low energy threshold, for example, a liquid scintillator neutrino detector. We note that even with a liquid scintillator detector, the dominant neutrino interaction is still IBD (Scholberg 2012).

If such a measurement was made, and there is not a significant amount of rotation (see the discussion on rotation in Section 5.3), one can immediately infer the gravitational binding energy of the remnant, since neutrinos carry away the vast majority $\left(\sim 99 \%{ }^{8}\right)$ of the gravitational binding energy. For typical nuclear EOS like the ones considered here, this results in a one-to-one mapping of the released gravitational binding energy to the baryonic mass of the remnant, and, hence, the gravitational mass of the remnant. This is most easily seen by fitting the gravitational binding energy of a cold $(T=0.1 \mathrm{MeV})$, neutrino-less $\beta$-equilibrium, non-rotating neutron star to its baryonic mass. From cold neutron star TOV solutions using the LS220 EOS one can obtain an empirical fit to better than $3 \%$ above a baryonic mass of $1.15 M_{\odot}$,

$$
E_{\text {binding }} \sim 1.12 \times 10^{53}\left(M_{\text {bary }} / M_{\odot}\right)^{2} \text { erg. }
$$

A similar fit for the HShen EOS gives

$$
E_{\text {binding }} \sim 9.78 \times 10^{52}\left(M_{\text {bary }} / M_{\odot}\right)^{2} \mathrm{erg},
$$

and is accurate to $5 \%$ above baryonic masses of $1.15 M_{\odot}$. Below $M_{\text {bary }}=1.15 M_{\odot}$, the empirical quadratic fit is not as

\footnotetext{
8 The remaining $\sim 1 \%$ of the energy is predominantly shared among the kinetic energy of the explosion, the original binding energy of the unbound stellar mantle, and the binding energy of the iron core at the onset of collapse.
}

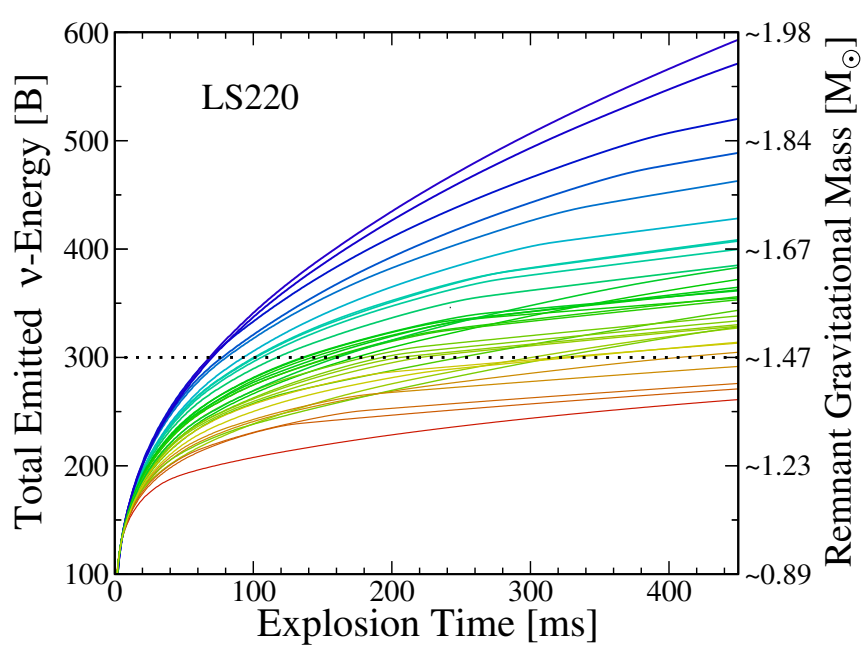

Figure 6. Total emitted neutrino energy estimated from the enclosed baryonic mass as a function of explosion time for the LS220 EOS. A measurement of the total emitted neutrino energy and an estimate of the explosion time constrains progenitor structure. For reference, we provide the cold neutron star gravitational mass associated with the released binding energy on the right ordinate. This figure is constructed using a fit of the gravitational binding energy of a cold neutron star to its baryonic mass, $E_{v}^{\text {total }} \sim 1.12 \times 10^{53}\left(M_{\text {bary }} / M_{\odot}\right)^{2} \mathrm{erg}$, and the baryonic mass enclosed in the shock at any given time. This defines the explosion time to be, in a Lagrangian sense, the time at which the outermost final neutron star mass element accretes through the shock. The color coding corresponds to $\xi_{1.75}$, and the color coding is provided in Figure 3.

(A color version of this figure is available in the online journal.)

accurate. However, all models considered here reach a baryonic protoneutron star mass of $1.15 M_{\odot}$ within $\sim 10 \mathrm{~ms}$ of bounce. Hence, we believe that the above fits are acceptable for the iron-core core collapse events considered here.

We now make the assumption that an explosion launched at a particular postbounce time will result in a neutron star remnant with a baryonic mass equal to the baryonic mass that has accreted through the shock up until the time of the explosion. This neglects any late-time fallback of material onto the protoneutron star which would lead to additional neutrino emission. Fryer (2009) and Ugliano et al. (2012) predict fallback masses $\lesssim 5 \%-10 \%$ of the initial protoneutron star remnant mass. We also neglect any asymmetric mass accretion that may occur in the early explosion phase. In Figure 6, we convert the baryonic mass enclosed by the shock to the total emitted neutrino energy using Equations (14) and (15). We plot this for all progenitor models (run with the LS220 EOS) as a function of the hypothetical time of explosion. As a concrete example, consider the situation where $300 \mathrm{~B}$ (shown as the dashed line in Figure 6) of total neutrino energy was observational inferred. This could correspond (1) to a progenitor with a high compactness parameter that exploded at an early time, e.g., model s40WH07 at $70 \mathrm{~ms}$ or (2) to a low progenitor with low compactness parameter that exploded at late times, e.g., model s12WH07 at $400 \mathrm{~ms}$. If we have an estimate of the explosion time, e.g., via characteristic features in the neutrino observables, then we can use the combined measurement to probe the progenitor core structure. This is further quantified in Figure 7, where we choose three total emitted neutrino energies, 250,300 , and $350 \mathrm{~B}$, and determine the time at which the explosion must have been launched for a given compactness and the respective total emitted energy. We plot this explosion time versus $\xi_{1.75}$ for all models and both EOS. In general, for a fixed total emitted energy, as the compactness parameter of 


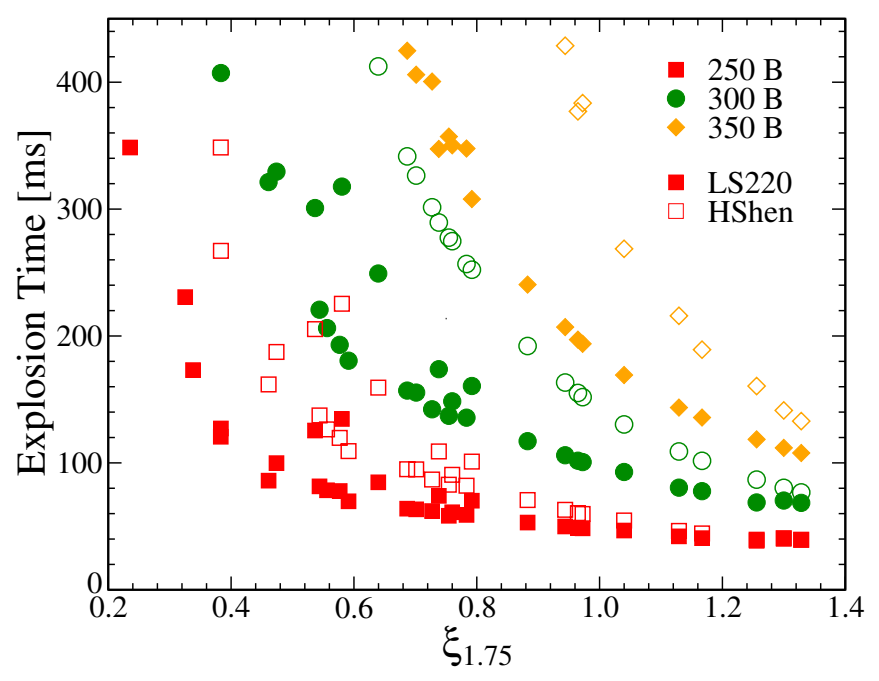

Figure 7. Explosion time for various progenitors and EOS, under the constraint of a fixed amount of released neutrino energy. We show three values of the total released energy, 250, 300, and $350 \mathrm{~B}$. Progenitors with high compactness must explode at earlier times to achieve the same total amount of released energy in neutrinos when compared to a progenitor with a lower compactness. The strong EOS dependence is due to the different binding energies resulting from the two EOS. The LS220 EOS leads to a more compact neutron star, releasing 14\% energy for a given baryonic mass.

(A color version of this figure is available in the online journal.)

the progenitor increases, the explosion time must decrease. For progenitors with high $\xi_{1.75}(\gtrsim 0.8)$, there is a clear mapping between the explosion time and the compactness parameter, given a specific total emitted neutrino energy and an EOS. As was the case for the total IBD rates, there is some scatter at low $\xi_{1.75}(\lesssim 0.8)$, but there is still an overall trend.

Figure 7 shows that there is a very strong EOS dependence. While the baryonic mass inside the shock as a function of postbounce time does not vary strongly with EOS, the gravitational binding energy released does. Based on the above empirical fits to the gravitational binding energy, the LS220 EOS leads to $\sim 14 \%$ more energy release than the HShen EOS for the same baryonic mass. This method of determining the compactness parameter (by combining an estimate of the explosion time and the total emitted neutrino energy) can be used together with the methods described above (using the postbounce, pre-explosion IBD rates) as a consistency check, or to break degeneracies, which we will discuss next in Section 5.3.

\subsection{Degeneracies in Neutrino Observables}

There are a number of degeneracies and uncertainties that may prevent fully conclusive statements regarding the mapping from detected signal to progenitor core structure. These include nuclear EOS, rotation, viewing angle, distance, and neutrino oscillations (including collective oscillations). We will discuss each one of these consecutively and independently, although all may be relevant in a generic situation.

Nuclear EOS. We have already briefly explored the dependence of the neutrino observables on the nuclear EOS by taking two very different EOS and comparing the emitted neutrino signal. The total number of interactions predicted to be detected from a given progenitor model varies with EOS (cf. Figure 5) in such a way that a high compactness model paired with the HShen EOS produces the same number of interactions as a model with a slightly lower compactness paired with a softer EOS, like the LS220. Hence, there is a clear degeneracy between the pro-

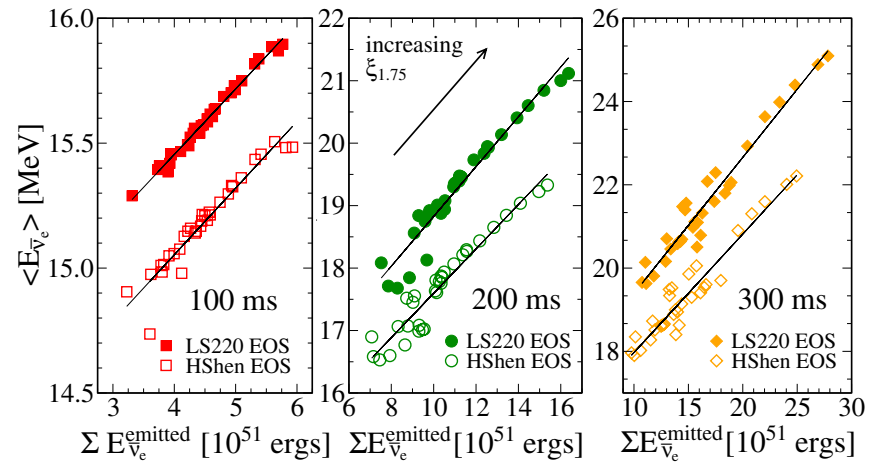

Figure 8. Average $\bar{v}_{e}$ energy vs. cumulative emitted $\bar{v}_{e}$ energy each EOS and progenitor. This is shown for three postbounce times: $100 \mathrm{~ms}$ (left panel), $200 \mathrm{~ms}$ (center panel), and $300 \mathrm{~ms}$ (right panel). Solid (open) points correspond to the LS220 (HShen) EOS. The degeneracy between EOS and cumulative $\bar{v}_{e}$ energy is broken when the average energy is taken into account. We find that it is a robust prediction across all progenitors and postbounce pre-explosion times.

(A color version of this figure is available in the online journal.)

genitor compactness and the EOS. However, a water Cherenkov detector will not only detect a given number of IBD interactions, but also their energy distribution. From this distribution, and the detector response, one can work backward to reconstruct both the emitted average $\bar{v}_{e}$ energy and the luminosity. We show in Figure 8 how knowledge of the emitted energy spectrum can break the degeneracy with cumulative emitted $\bar{v}_{e}$ energy and EOS, provided the distance to the core collapse event is known (see the discussion of distance uncertainties later in this section). For each model and EOS, we plot the average $\bar{v}_{e}$ energy as a function of cumulative emitted $\bar{v}_{e}$ energy at three select postbounce times: 100, 200, and $300 \mathrm{~ms}$. The data from each of the 32 progenitors fall on a unique line that is parameterized by the compactness. In principle, provided very large statistics and ultimate predictions of neutrino observables, this allows one to break the EOS-progenitor degeneracy between EOS. In reality, achieving an instantaneous measurement of the neutrino average energy to within a few percent will be difficult, and there will likely be a large suite of nuclear EOS that could reproduce the observables within error.

Rotation and viewing angle. The effect of rotation on the neutrino signal may be more difficult to disentangle from the effects of progenitor structure. To explore the effect of rotation, we perform 1.5D simulations of rotating core collapse. These simulations treat rotation in a spherically symmetric way, using "shellular rotation" (Thompson et al. 2005; O'Connor $\&$ Ott 2010). This approximation only captures the spherically averaged centrifugal effect of rotation on the matter. As the amount of rotation is increased, the protoneutron star becomes more and more centrifugally supported. Increased rotation leads to lower densities and temperatures throughout the protoneutron star. This in turn effects the neutrino signal. In Figure 9, we plot the $\bar{v}_{e}$ luminosities and average $\bar{v}_{e}$ energies determined from nuGR1D for 12 rotating core collapse simulations. We use model $s 15 \mathrm{WH} 07$ paired with the LS220 EOS. The initial rotation rate is assigned via (O'Connor \& Ott 2011)

$$
j(r)=j_{16, \infty}\left[1+\left(\frac{A_{M_{\odot}}}{r}\right)^{2}\right]^{-1} \times 10^{16} \mathrm{~cm}^{2} \mathrm{~s}^{-1},
$$

where $A_{M_{\odot}}$ is the radius that encloses $1 M_{\odot}$, which for the $s 15 \mathrm{WH} 07$ progenitor is $703 \mathrm{~km}$, and $j_{16, \infty}$ is the specific angular momentum at infinity, in units of $10^{16} \mathrm{~cm}^{2} \mathrm{~s}^{-1}$. 


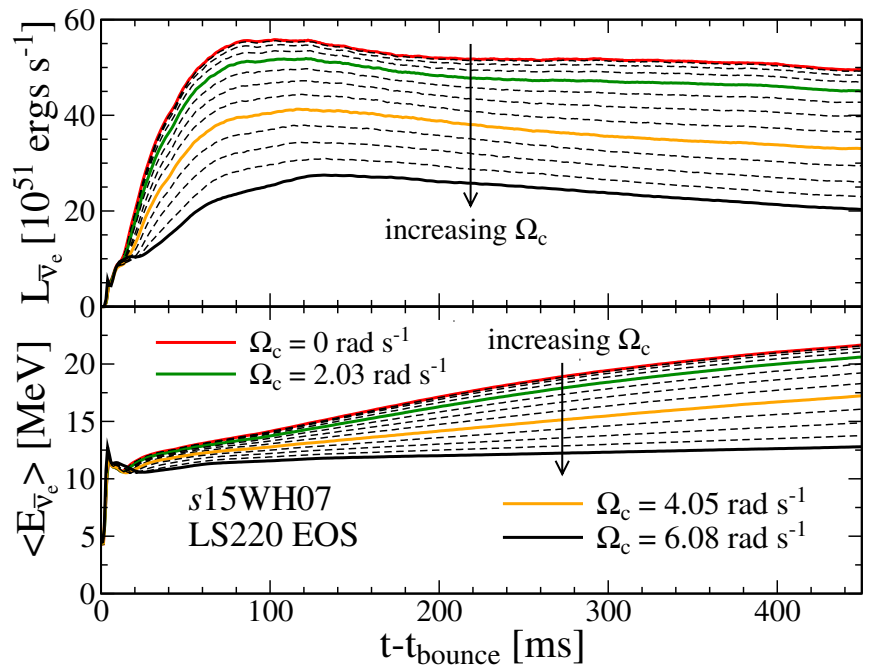

Figure 9. Luminosities (top panel) and average energies (bottom panel) from $1.5 \mathrm{D}$ rotating core collapse simulations of model $s 15 \mathrm{WH} 07$ for various initial central angular velocities. We assign angular momentum to the $s 15 \mathrm{WH} 07$ progenitor via Equation (16) with values of $j_{16, \infty}$ ranging from 0 to 3 in 0.25 increments. This gives initial central rotation rates of 0 to $\sim 6.08 \mathrm{rad} \mathrm{s}^{-1}$ in increments of $\sim 0.51 \mathrm{rad} \mathrm{s}^{-1}$. In our simulations, increased rotation lowers both the neutrino luminosity and the average energy.

(A color version of this figure is available in the online journal.)

We vary $j_{16, \infty}$ from 0 to 3 in increments of 0.25 . For the $s 15 \mathrm{WH} 07$ progenitor, the initial central angular velocity is $\Omega_{c}=2.03 j_{16, \infty} \mathrm{rad} \mathrm{s}^{-1}$, giving a range of $\Omega_{c}$ from 0 to $6.08 \mathrm{rad} \mathrm{s}^{-1}$. At bounce, the $j_{16, \infty}=1, \Omega_{c}=2.03 \mathrm{rad} \mathrm{s}^{-1}$ model has a rotation period of $\sim 2.4 \mathrm{~ms}$, which decreases nearly linearly with increasing initial $j_{16, \infty}$. Increased rotation leads to the overall reduction of both the $\bar{v}_{e}$ luminosity and average energy. For example, with $\Omega_{c}=0,2.03,4.05$, and $6.08 \mathrm{rad} \mathrm{s}^{-1}$ the $\bar{v}_{e}$ luminosity at $100 \mathrm{~ms}$ is $\sim 55, \sim 52, \sim 40$, and $\sim 25 \mathrm{~B} \mathrm{~s}^{-1}$, respectively. While we do not show the $v_{e}$ and $v_{x}$ luminosities and average energies, they follow the $\bar{v}_{e}$ trends. The only exception to this is the $v_{e}$ neutronization burst where rotation in our 1.5D approach does not significantly alter the $v_{e}$ luminosity. Our 1.5D approach to rotation does not capture the anisotropy of the neutrino emission, rather just the angle-averaged value. Ott et al. (2008) considered a model with an initial rotation rate of $\pi$ $\mathrm{rad} \mathrm{s}^{-1}$, roughly corresponding to our $j_{16, \infty}=1.5$ model. They found that the ratio of polar to equatorial luminosity can be as large as three to four and that average neutrino energies in polar regions can be harder by $\gtrsim 1-2 \mathrm{MeV}$ than on the equator. We find that it is not possible with the cumulative neutrino signals alone to break the degeneracy between rotation and the progenitor star-EOS combinations. The angle-averaged $\bar{v}_{e}$ luminosity and average energy from a rotating system mimic the $\bar{v}_{e}$ luminosity and average energy from a non-rotating system with a progenitor with lower compactness and/or a stiffer EOS. In addition, the rotational energy stored in the neutron star remnant can lead to an underestimate of the total emitted neutrino energy, again mimicking a lower-compactness progenitor and/or a stiffer EOS. However, all hope is not lost: stellar evolution and current constraints on pulsar birth spins (e.g., Heger et al. 2005; Ott et al. 2006) suggest that most massive stars will be spinning too slowly for rotation to have a strong effect on dynamics and neutrino signal. Heger et al. (2005), for example, predict a presupernova rotation rate of $0.2 \mathrm{rad} \mathrm{s}^{-1}$ for a $15 M_{\odot}$ star, which is smaller than all rotation rates considered here. If, on the other hand, the progenitor is rapidly spinning, the degeneracy in the neutrino signal may be broken by coincident observations in gravitational waves that are able to constrain the rotation rate of the collapsed core (Ott et al. 2012).

Distance. If the electromagnetic signal is blocked, by, for example, the galactic center, we may not be able to obtain a reliable distance to the supernova. The $\bar{v}_{e}$ flux at Earth follows an inverse square law, while the spectral distribution will not change over the distances of relevance. This means that degeneracy with distance is also hard to break with the IBD neutrino signal alone for the following reason: a highcompactness progenitor at a large distance can produce the same energy-integrated flux at Earth as a low-compactness progenitor. Less neutrinos are emitted in the latter but the flux has not been diluted as much due to the closer distance. If the nuclear EOS is not known, the observed neutrino flux and energy spectra could be associated with a range of nuclear EOS-distance combinations. A progenitor at a large distance could produce the same neutrino flux and energy distribution as a lower-compactness progenitor with a softer EOS. Here, the softer EOS, which will give rise to an increased average energy, compensates for the decrease in the average energy produced by the smaller compactness.

Future constraints on the nuclear EOS could break this degeneracy since one could more reliably associate an observed energy distribution with a particular progenitor compactness (Figure 3). This may even provide a distance estimate, as would a detection of the neutronization burst signal (Kachelrieß et al. 2005). It does not show a strong dependence on the progenitor model, but is difficult to detect since the $v_{e}$ cross sections in water Cherenkov detectors are much lower than the IBD cross section, resulting in few interactions. Some detector materials have significantly larger $v_{e}$ cross sections and would be better suited to detect the neutronization burst, such as liquid argon (Scholberg 2012) and lead (Duba et al. 2008).

Neutrino oscillations. First, considering only matter-induced neutrino oscillations, in the normal mass hierarchy, the electron antineutrino signal at Earth is a composite spectrum of $\cos ^{2}\left(\theta_{12}\right) \sim 70 \%$ of the original $\bar{v}_{e}$ neutrinos and $\sin ^{2}\left(\theta_{12}\right) \sim$ $30 \%$ of the original $\bar{v}_{x}$ spectrum, where $\theta_{12}$ is the mixing angle between the mass eigenstates 1 and 2 . In the inverted neutrino mass hierarchy, the entire $\bar{v}_{e}$ signal is replaced with the original $\bar{v}_{x}=v_{x}$ signal (Dighe \& Smirnov 2000). In either case, we still expect the total number of IBD interactions to increase with the compactness parameter of the progenitor since both the $\bar{v}_{e}$ and the $v_{x}$ luminosity increase. If the hierarchy of the neutrino mass eigenstates is not determined by neutrino experiments before the next nearby core-collapse supernova, the early postbounce, pre-explosion neutrino luminosities may provide an answer (Kachelrieß et al. 2005; Serpico et al. 2012). This relies on the systematically different rise times of the $v_{x}$ and $\bar{v}_{e}$ signals. We also point out a degeneracy in disentangling the spectral properties of the emitted neutrino spectra from the matter-induced oscillated neutrino spectra observed in Earth detectors (Minakata et al. 2008).

Much more troublesome are the collective neutrino oscillations that arise from coherent neutrino-neutrino forward scattering. Collective oscillations are very sensitive to the energy spectra (both the distribution and magnitude) of all neutrino flavors and the background matter density. Since the governing equations are highly nonlinear, there are currently no simple analytic expressions predicting the neutrino signal at Earth based on the output of core-collapse simulations. Recent studies suggest that during the early postbounce, pre-explosion phase, 
collective neutrino oscillations may be suppressed (Chakraborty et al. 2011a, 2011b; Sarikas et al. 2012); however, the community has not yet reached consensus, see, e.g., Cherry et al. (2012) and Dasgupta et al. (2012).

\section{DISCUSSION}

The next nearby core-collapse supernova will be extremely well observed in neutrinos. Super-Kamiokande alone will observe $\sim 7000$ electron antineutrinos from a typical core-collapse supernova at a fiducial galactic distance of $10 \mathrm{kpc}$. Future detectors of the scale of the proposed hyper-Kamiokande may see in excess of $10^{5}$ interactions. Such high-statistics observations will provide rich information on the neutrino signal. Comparison with theoretical model predictions will allow to falsify or constrain a broad range of hypotheses in core-collapse supernova astrophysics and nuclear/neutrino physics. Unexpected signal features may lead to the discovery of new physics.

In this study, our focus has been on the imprint of the progenitor star's structure on the neutrino signal in the postbounce pre-explosion phase of core-collapse supernovae. We have carried out a large set of spherically symmetric radiationhydrodynamics simulations of core collapse and the early postbounce phase with the goal of studying trends in the neutrino signal with variations in progenitor structure.

Our results show, in agreement with previous work (e.g., Burrows \& Mazurek 1983; Liebendörfer et al. 2001, 2002, 2003, 2004; Thompson et al. 2003; Kachelrieß et al. 2005; Buras et al. 2006; Serpico et al. 2012), that the $v_{e}$ signal from the neutronization burst emerging shortly after bounce has very little progenitor dependence, due to the universal nature of homologous inner core collapse.

The neutrino signal in the postbounce pre-explosion phase is determined primarily by the accretion luminosity of outer iron core, silicon shell, and oxygen shell material. The postbounce accretion rate depends on the inner structure of the progenitor at the presupernova stage. Our results show that the pre-explosion neutrino signal has an essentially monotonic dependence on progenitor structure described by a single parameter, the compactness $\xi_{M} \propto M / R(M)$ (where $M$ is a typical baryonic mass reaching the center over the timescale of interest). The greater a progenitor's $\xi_{M}$, the higher are the emitted luminosities and average energies of all neutrino species. Scaling in the same way is the total emitted energy in neutrinos over the entire protoneutron star cooling phase for a given explosion time at which accretion is shut off. These trends are robust and independent of the nuclear EOS. They are also rather insensitive to the particular choice of the reference mass $M$ in $\xi_{M}$ as long as it is in the range of typical neutron star baryonic masses $\left(\sim 1.4-2.5 M_{\odot}\right)$ and we find $\xi_{1.75}$ to be a good choice.

The monotonic dependence of the pre-explosion neutrino emission on progenitor compactness translates directly to the neutrino signal observed by detectors, provided collective neutrino oscillations do not lead to complicated swaps of flavor spectra that brake the dependence of the observed signal on progenitor structure. Neutrino observations of the next nearby core collapse event thus may, in principle, allow quantitative constraints on the inner structure of the progenitor star. As an example with real neutrino data, we consider the early postbounce neutrino signal observed from SN 1987A by the Kamiokande-II experiment (Hirata et al. 1987). Of the 11 interactions that were observed, the first four occurred within $323 \mathrm{~ms}$ of each other. All interactions observed by Kamiokande-II are consistent with being IBD interactions (Hirata et al. 1987). We assume that the

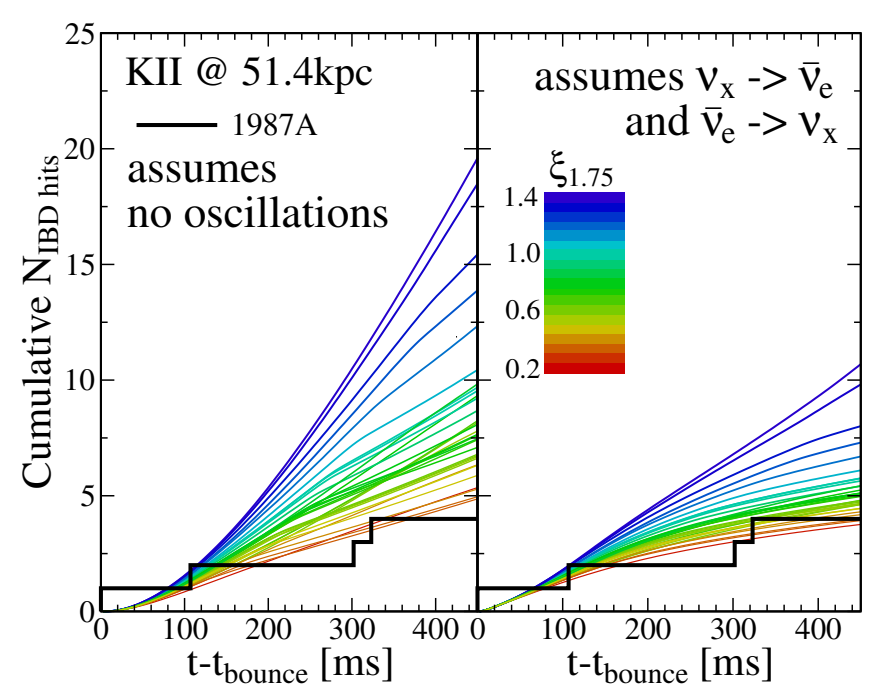

Figure 10. Predictions of the cumulative IBD interactions in Kamiokande-II for a core-collapse event at $51.4 \mathrm{kpc}$. The left panel assumes no neutrino oscillations, while the right panel assumes a swapping of the $\bar{v}_{e}$ and $v_{x}$ spectra. The colorcoded lines denote different progenitors and are generated with SNOwGLoBES based on our simulations with the LS220 EOS. More details are provided in the text. We overlay the cumulative detected interactions observed in Kamiokande-II from SN 1987A, assuming the first interaction denotes the time of core bounce. We caution the reader by noting that the very low number statistics make it hard to draw any conclusions regarding the progenitor of SN1987A.

(A color version of this figure is available in the online journal.)

first interaction occurs at the onset of the postbounce phase, although the actual bounce time is likely somewhat earlier. In Figure 10, we plot the cumulative number of detected interactions observed from SN 1987A in the first $500 \mathrm{~ms}$ along with the SNOwGLoBES prediction from our simulations with the LS220 EOS using a $2.14 \mathrm{kT}$ water Cherenkov detector at $51.4 \mathrm{kpc}$. We use the efficiencies quoted in Burrows (1988) and the smearing matrices from the SNOwGLoBES detector configuration wc100kt30prct. To show the full range of the possible effects of MSW neutrino oscillations, we show the expected number of interactions assuming no oscillations and assuming a complete switch of the $\bar{v}_{e}$ and $v_{x}$ spectra (as would be the case in the inverted mass hierarchy; the normal hierarchy is a combination of these two signals). During the accretion phase the expected number of detected interactions from an oscillated $v_{x}$ spectrum can be significantly smaller than the electron-type neutrino luminosity due to the smaller neutrino luminosity in the accretion phase. While the quantitative results obviously depend on neutrino oscillation details, the qualitative trend with $\xi_{M}$ is unbroken. Comparing our predictions with the interactions observed from SN 1987A one notes (but must keep the very small-number statistics in mind) that either the explosion must have occurred early in the postbounce phase and/or the progenitor must have had a relatively low $\xi_{1.75}$. Stated another way, that data strongly disfavor a late-time explosion in a high compactness model. Both of these statements are broadly consistent with the previous work of Bruenn (1987) and Burrows (1988). It is also not inconsistent with the proposed ZAMS mass of $\sim 18-20 M_{\odot}$ for Sanduleak -69 202 (e.g., Woosley et al. 2002), the blue supergiant progenitor star of SN 1987A. However, the mapping between ZAMS mass and stellar structure (i.e., compactness) at the presupernova stage appears to be highly nonmonotonic (cf. Figure 1; Woosley et al. 2002; Woosley \& Heger 2007). This makes it very difficult to link the observed neutrino 
signal to ZAMS mass without additional constraints from classical astronomical observations in the electromagnetic spectrum.

In the discussion of SN 1987A, we have ignored uncertainties regarding nuclear EOS, rotation of the progenitor core, distance, and collective neutrino oscillations. All affect the pre-explosion neutrino signature in ways that may be degenerate with variations in the compactness parameter. However, as we have shown, large variations in the stiffness of the nuclear EOS in the density and temperature regime relevant in the pre-explosion phase can be disentangled from $\xi_{M}$ via observations of the emitted neutrino spectrum. Rapid rotation, which decreases both (angleaveraged) luminosities and average neutrino energies, can be constrained by gravitational wave observations of a galactic event (e.g., Ott et al. 2012). Distance uncertainties from electromagnetic observations, which translate into uncertainties in the absolute luminosities, can be reduced by exploiting the generic neutronization burst as a standard candle (Kachelrieß et al. 2005). Collective neutrino oscillations are not yet fully understood and have not been directly incorporated in neutrino radiation-hydrodynamics simulations. They may lead to one or multiple energy-dependent swaps of spectra between flavors and could thus complicate the mapping between neutrino signal and compactness parameter. However, the recent understanding suggests that collective oscillations may not be significant in the pre-explosion phase (Chakraborty et al. 2011b, 2011a; Sarikas et al. 2012; but see also Cherry et al. 2012 and Dasgupta et al. 2012).

Our goal with this study was to highlight overall trends of the pre-explosion neutrino signal with progenitor structure in the limit of spherical symmetry. We expect these overall trends to be robust and to carry over to the multi-dimensional case. We did not aim at making precise and robust quantitative predictions for any individual model. These are not possible with our current spherically symmetric radiation-hydrodynamics treatment, which neglects inelastic scattering, redshift, and velocitydependent terms (e.g., Lentz et al. 2012a, 2012b). Rotation is included only in an angle-averaged manner and other important multi-dimensional dynamics, in particular convection and the standing-accretion shock instability (Buras et al. 2006; Ott et al. 2008, 2012; Marek \& Janka 2009; Marek et al. 2009; Lund et al. 2010; Brandt et al. 2011), cannot be captured. Future work to remove these limitations will be necessary to produce the reliable signal predictions necessary for drawing quantitative conclusions from neutrino observations of the next nearby core-collapse supernova. However, even with fully realistic and complete simulation codes, large parameter studies will be necessary to account for prevailing uncertainties in the nuclear EOS and/or neutrino interaction physics.

We acknowledge helpful discussions with and input from John Beacom, Adam Burrows, Luc Dessart, Ken Nomoto, Ryan Patterson, Kate Scholberg, Mark Vagins, and Stan Woosley. C.D.O. thanks the Kavli Institute for the Physics and Mathematics of the Universe for hospitality while work on a draft of this article was carried out. The computations were performed at Caltech's Center for Advanced Computing Research on the cluster "Zwicky" funded through NSF grant No. PHY-0960291 and the Sherman Fairchild Foundation. Furthermore, computations were performed on Louisiana Optical Network Infrastructure computer systems under allocation loni_numrel06, on the NSF XSEDE Network under allocation TG-PHY100033, and on resources of the National Energy Research Scientific Computing Center, which is supported by the Office of
Science of the U.S. Department of Energy under Contract No. DE-AC02-05CH11231. EOC is supported in part by a postgraduate fellowship from the Natural Sciences and Engineering Research Council of Canada (NSERC). This research is supported in part by the National Science Foundation under grant Nos. AST-0855535 and OCI-0905046, by the Alfred P. Sloan Foundation, and by the Sherman Fairchild Foundation.

\section{REFERENCES}

Abbasi, R., et al. (IceCube Collaboration) 2011, A\&A, 535, A109 Aglietta, M., Alpat, B., Alyea, E. D., et al. 1992, NCimA, 105, 1793

Alekseev, E. N., Alekseeva, L. N., Volchenko, V. I., \& Krivosheina, I. V. 1987, JETPL, 45, 589

Alimonti, G., et al. (BOREXINO Collaboration) 2009, NIMPA, 600, 568

An, F. P., Bai, J. Z., Balantekin, A. B., et al. 2012, PhRvL, 108, 171803

Arnett, W. D., Bahcall, J. N., Kirshner, R. P., \& Woosley, S. E. 1989, ARA\&A, 27,629

Audit, E., Charrier, P., Chièze, J., \& Dubroca, B. 2002, arXiv:0206281

Beacom, J. F., \& Vogel, P. 1999, PhRvD, 60, 033007

Beck, A., Beroz, F., Carr, R., et al. 2011, SNOwGLoBES, revision 1373, http://www.phy.duke.edu/ schol/snowglobes/

Bionta, R. M., Blewitt, G., Bratton, C. B., Casper, D., \& Ciocio, A. 1987, PhRvL, 58,1494

Brandt, T. D., Burrows, A., Ott, C. D., \& Livne, E. 2011, ApJ, 728, 8

Bruenn, S. W. 1985, ApJS, 58, 771

Bruenn, S. W. 1987, PhRvL, 59, 938

Buras, R., Janka, H.-T., Rampp, M., \& Kifonidis, K. 2006, A\&A, 457, 281

Burrows, A. 1987, PhT, 40, 28

Burrows, A. 1988, ApJ, 334, 891

Burrows, A., Dessart, L., Livne, E., Ott, C. D., \& Murphy, J. 2007, ApJ, 664,416

Burrows, A., Klein, D., \& Gandhi, R. 1992, PhRvD, 45, 3361

Burrows, A., \& Lattimer, J. M. 1986, ApJ, 307, 178

Burrows, A., \& Mazurek, T. L. 1983, Natur, 301, 315

Burrows, A., Reddy, S., \& Thompson, T. A. 2006, NuPhA, 777, 356

Cadonati, L., Calaprice, F. P., \& Chen, M. C. 2002, APh, 16, 361

Cernohorsky, J., \& Bludman, S. A. 1994, ApJ, 433, 250

Chakraborty, S., Fischer, T., Mirizzi, A., Saviano, N., \& Tomàs, R. 2011a, PhRvD, 84, 025002

Chakraborty, S., Fischer, T., Mirizzi, A., Saviano, N., \& Tomàs, R. 2011b, PhRvL, 107, 151101

Cherry, J. F., Carlson, J., Friedland, A., Fuller, G. M., \& Vlasenko, A. 2012, PhRvL, 108, 261104

Dasgupta, B., \& Beacom, J. F. 2011, PhRvD, 83, 113006

Dasgupta, B., \& Dighe, A. 2008, PhRvD, 77, 113002

Dasgupta, B., O'Connor, E. P., \& Ott, C. D. 2012, PhRvD, 85, 065008

Davies, G. S. (for the NOvA Collaboration) 2011, arXiv: 1110.0112

Demorest, P. B., Pennucci, T., Ransom, S. M., Roberts, M. S. E., \& Hessels, J. W. T. 2010, Natur, 467, 1081

Dighe, A. S., \& Smirnov, A. Y. 2000, PhRvD, 62, 033007

Duan, H., Fuller, G. M., Carlson, J., \& Qian, Y.-Z. 2007, PhRvD, 75, 125005

Duan, H., Fuller, G. M., \& Qian, Y.-Z. 2010, ARNPS, 60, 569

Duba, C. A., Duncan, F., Farine, J., et al. 2008, JPhCS, 136, 042077

Einfeldt, B. 1988, in 16th Proc. Int Symp., Shock Tubes and Waves, Aachen, Germany, 1987 July 26-31 (Weinheim, Germany: VCH Verlag), 671

Fischer, T., Martínez-Pinedo, G., Hempel, M., \& Liebendörfer, M. 2012, PhRvD, 85,083003

Fischer, T., Whitehouse, S. C., Mezzacappa, A., Thielemann, F.-K., \& Liebendörfer, M. 2009, A\&A, 499, 1

Fischer, T., Whitehouse, S. C., Mezzacappa, A., Thielemann, F.-K., \& Liebendörfer, M. 2010, A\&A, 517, A80

Fogli, G., Lisi, E., Marrone, A., \& Tamborra, I. 2009, JCAP, JCAP04(2009)30

Fryer, C. L. 2009, ApJ, 699, 409

Fukuda, S., Fukuda, Y., Hayakawa, T., et al. 2003, NIMPA, 501, 418

Halzen, F., \& Raffelt, G. G. 2009, PhRvD, 80, 087301

Hannestad, S., Raffelt, G. G., Sigl, G., \& Wong, Y. Y. Y. 2006, PhRvD, 74, 105010

Hebeler, K., Lattimer, J. M., Pethick, C. J., \& Schwenk, A. 2010, PhRvL, 105,161102

Heger, A., Woosley, S. E., \& Spruit, H. C. 2005, ApJ, 626, 350

Hirata, K., Kajita, T., Koshiba, M., Nakahata, M., \& Oyama, Y. 1987, PhRvL, 58,1490

Huber, P., Kopp, J., Lindner, M., Rolinec, M., \& Winter, W. 2007, CoPhC, 177,432 
Huber, P., Lindner, M., \& Winter, W. 2005, CoPhC, 167, 195

Hüdepohl, L., Müller, B., Janka, H.-T., Marek, A., \& Raffelt, G. G. 2010, PhRvL, 104,251101

Ikeda, M., et al. (Super-Kamiokande Collaboration) 2007, ApJ, 669, 519

Janka, H.-T., Langanke, K., Marek, A., Martínez-Pinedo, G., \& Müller, B. 2007, $\mathrm{PhR}, 442,38$

Jegerlehner, B., Neubig, F., \& Raffelt, G. 1996, PhRvD, 54, 1194

Kachelrieß, M., Tomàs, R., Buras, R., et al. 2005, PhRvD, 71, 063003

Kotake, K. 2011, CRPhy, submitted, arXiv:1110.5107

Kraus, C., \& Peeters, S. J. M. 2010, PrPNP, 64, 273

Kuroda, T., Kotake, K., \& Takiwaki, T. 2012, ApJ, 755, 11

Lattimer, J. M., \& Swesty, F. D. 1991, NuPhA, 535, 331

Lentz, E. J., Mezzacappa, A., Bronson Messer, O. E., Hix, W. R., \& Bruenn, S. W. 2012a, ApJ, 760, 94

Lentz, E. J., Mezzacappa, A., Bronson Messer, O. E., et al. 2012b, ApJ, 747, 73

Liebendörfer, M. 2005, ApJ, 633, 1042

Liebendörfer, M., Messer, O. E. B., Mezzacappa, A., et al. 2004, ApJS, 150,263

Liebendörfer, M., Messer, O. E. B., Mezzacappa, A., \& Hix, W. R. 2001, in AIP Conf. Ser. 586, 20th Texas Symposium on Relativistic Astrophysics, ed. J. C. Wheeler \& H. Martel (Melville, NY: AIP), 472

Liebendörfer, M., Messer, O. E. B., Mezzacappa, A., et al. 2002, in Proc. 11th Workshop on Nuclear Astrophysics, ed. W. Hillebrandt \& E. G. M. Müller (München, Germany: Max-Planck-Institut für Astrophysik), 126

Liebendörfer, M., Mezzacappa, A., Messer, O. E. B., et al. 2003, NPA, 719, 144

Liebendörfer, M., Rampp, M., Janka, H.-T., \& Mezzacappa, A. 2005, ApJ, 620,840

Loredo, T. J., \& Lamb, D. Q. 2002, PhRvD, 65, 063002

Lund, T., Marek, A., Lunardini, C., Janka, H.-T., \& Raffelt, G. 2010, PhRvD, 82, 063007

Marek, A., \& Janka, H.-T. 2009, ApJ, 694, 664

Marek, A., Janka, H.-T., Buras, R., Liebendörfer, M., \& Rampp, M. 2005, A\&A, 443, 201

Marek, A., Janka, H.-T., \& Müller, E. 2009, A\&A, 496, 475

Mayle, R., Wilson, J. R., \& Schramm, D. N. 1987, ApJ, 318, 288

Mikheev, S., \& Smirnov, A. 1986, NCimN, 9, 17

Minakata, H., Nunokawa, H., Tomàs, R., \& Valle, J. W. F. 2008, JCAP, JCAP12(2008)006

Minerbo, G. N. 1978, JQSRT, 20, 541

Müller, B., Janka, H.-T., \& Heger, A. 2012a, ApJ, submitted (arXiv:1205.7078)

Müller, B., Janka, H.-T., \& Marek, A. 2012b, ApJ, 756, 84

O’Connor, E., \& Ott, C. D. 2010, CQGra, 27, 114103

O'Connor, E., \& Ott, C. D. 2011, ApJ, 730, 70

Ott, C. D. 2009, CQGra, 26, 063001

Ott, C. D., Abdikamalov, E., O’Connor, E., et al. 2012, PhRvD, 86, 024026
Ott, C. D., Burrows, A., Dessart, L., \& Livne, E. 2008, ApJ, 685, 1069

Ott, C. D., Burrows, A., Thompson, T. A., Livne, E., \& Walder, R. 2006, ApJS, 164,130

Ott, C. D., O'Connor, E. P., \& Dasgupta, B. 2011, in Proc. Hamburg Neutrinos from Supernova Explosions (HAvSE) 2011 Conf., DESY Proc. Ser., 22

Özel, F., Baym, G., \& Güver, T. 2010, PhRvD, 82, 101301

Pagliaroli, G., Vissani, F., Coccia, E., \& Fulgione, W. 2009a, PhRvL, 103, 031102

Pagliaroli, G., Vissani, F., Costantini, M. L., \& Ianni, A. 2009b, APh, 31, 163

Pantaleone, J. T. 1992, PhLB, 287, 128

Piepke, A. 2001, NuPhS, 91, 99

Pons, J. A., Ibáñez, J. M., \& Miralles, J. A. 2000, MNRAS, 317, 550

Pontecorvo, B. 1968, JETP, 26, 984

Raffelt, G. G. 2010, PrPNP, 64, 393

Rampp, M., \& Janka, H.-T. 2002, A\&A, 396, 361

Roberts, L. F., Shen, G., Cirigliano, V., et al. 2012, PhRvL, 108, 061103

Sarikas, S., Raffelt, G. G., Hüdepohl, L., \& Janka, H.-T. 2012, PhRvL, 108 , 061101

Sato, K., \& Suzuki, H. 1987, PhRvL, 58, 2722

Scholberg, K. 2012, ARNPS, 62, 81

Serpico, P. D., Chakraborty, S., Fischer, T., et al. 2012, PhRvD, 85, 085031

Shen, H., Toki, H., Oyamatsu, K., \& Sumiyoshi, K. 2011, ApJS, 197, 20

Shibata, M., Kiuchi, K., Sekiguchi, Y., \& Suwa, Y. 2011, PThPh, 125, 1255

Steiner, A. W., Lattimer, J. M., \& Brown, E. F. 2010, ApJ, 722, 33

Sumiyoshi, K., Yamada, S., \& Suzuki, H. 2008, ApJ, 688, 1176

Thompson, T. A. 2002, PhD thesis, Univ. Arizona

Thompson, T. A., Burrows, A., \& Pinto, P. A. 2003, ApJ, 592, 434

Thompson, T. A., Quataert, E., \& Burrows, A. 2005, ApJ, 620, 861

Timmes, F. X., \& Arnett, D. 1999, ApJS, 125, 277

Tomas, R., Semikoz, D., Raffelt, G., Kachelriess, M., \& Dighe, A. 2003, PhRvD, 68, 093013

Ugliano, M., Janka, H.-T., Marek, A., \& Arcones, A. 2012, ApJ, 757, 69

van Leer, B. J. 1977, JCoPh, 23, 276

Vigorito, C. (LVD Collaboration) 2011, PuPhS, 221, 410

Wilson, J. R. 1985, in Numerical Astrophysics, ed. J. M. Centrella, J. M. Leblanc, \& R. L. Bowers (Boston, MA: Jones and Bartlett Publ.), 422

Wilson, J. R., Mayle, R., Woosley, S. E., \& Weaver, T. 1986, NYASA, 470,267

Wolfenstein, L. 1978, PhRvD, 17, 2369

Woosley, S. E., \& Heger, A. 2007, PhR, 442, 269

Woosley, S. E., Heger, A., \& Weaver, T. A. 2002, RvMP, 74, 1015

Woosley, S. E., \& Weaver, T. A. 1995, ApJS, 101, 181

Woosley, S. E., Wilson, J. R., \& Mayle, R. 1986, ApJ, 302, 19

Wurm, M., Beacom, J. F., Bezrukov, L. B., et al. 2012, APh, 35, 685

Yüksel, H., \& Beacom, J. F. 2007, PhRvD, 76, 083007 